\title{
SMOOTHING OF ISOLATED HYPERSURFACE SINGULARITIES AND QUILLEN METRICS*
}

\author{
KEN-ICHI YOSHIKAWA ${ }^{\dagger}$
}

\begin{abstract}
Using Bismut-Lebeau's theorem, we determine the singularity of Quillen metrics for smoothing of isolated hypersurface singularities and apply it to compute the asymptotic behavior of Ray-Singer analytic torsion for such degenerations.
\end{abstract}

0. Introduction. In [Q], Quillen introduced a metric on the determinant of cohomologies which is called Quillen metric today and calculated its curvature in some cases. After Quillen, in the celebrated work [B-G-S], Bismut-Gillet-Soulé generalized his result to arbitrary smooth morphism of Kähler manifolds and established the Riemann-Roch-Grothendieck formula at the differential form level. The purpose of this article is to study the boundary behavior of Quillen metrics. Namely, we investigate the behavior of Quillen metrics for a certain degenerating family of algebraic manifolds. Such a problem was treated by Wolpert ([W]) and also by Bismut-Bost ([B-B]) in the case of relative dimension one. (See also [T-T]). In [B 2], Bismut studies this problem when the family is ordinary singular and obtains a generalization of the result in [B-B]. We study the same problem for another type of degenerating family. When a family of complex manifold is given, the discriminant locus is the set of all points in the base space whose fiber is singular. In many families, generic point of the discriminant locus consists of those points whose fiber has only isolated hypersurface singularities. Therefore, it is natural to focus our consideration on the following degeneration.

Definition. Let $\pi: X \rightarrow S=\{t \in \mathbb{C} ;|t|<1\}$ be a proper surjective holomorphic map of complex manifolds. The family $(\pi, X, S)$ is said to be a smoothing of isolated hypersurface singularities (IHS), if $\pi$ is of maximal rank outside of finite number of points in $X_{0}:=\pi^{-1}(0)$. In particular, $X_{0}$ has only isolated hypersurface singularities.

To state our theorem, fix notations. Let $(\pi, X, S)$ be a smoothing of IHS, $g_{X}$ a Kähler metric of $X$ and $g_{X / S}$ the induced metric on $T X / S$. Let $(E, h)$ be a holomorphic Hermitian vector bundle over $X$. Let $\lambda(E)$ be the determinant of cohomologies and $\|\cdot\|_{Q}$ its Quillen metric relative to $g_{X / S}$ and $h$ (cf. $\S 1$ ).

Main Theorem. Suppose that $(\pi, X, S)$ is projective over $S$. Then, $\|\cdot\|_{Q}$ extends to a singular Hermitian metric whose curvature current is

$$
c_{1}\left(\lambda(E),\|\cdot\|_{Q}\right)=\frac{(-1)^{n+1}}{(n+2) !} r(E) \mu\left(X_{0}\right) \delta_{0}+\pi_{*}\left(T d\left(T X / S, g_{X / S}\right) \operatorname{ch}(E, h)\right)^{(1,1)}
$$

where $\operatorname{dim} X=n+1, r(E)=\operatorname{rank} E, \delta_{0}$ the Dirac measure supported at 0 and $\mu\left(X_{0}\right)$ the total Milnor number of the singular fiber. Moreover, continuous part of the curvature current, namely $\pi_{*}\left(T d\left(T X / S, g_{X / S}\right) \operatorname{ch}(E, h)\right)^{(1,1)}$, lies in $L_{l o c}^{p}(S)$ for some $p>1$ depending only on $\operatorname{Sing} X_{0}$.

* Received January 13, 1998; accepted for publication April 28, 1998. We are grateful to the Nishiaki Foundation for financial support and also to the Fourier Institute for its hospitality where the first version of this article was written. Finally, we express our thanks to professors J.-P. Demailly for helpful advices and J.-M. Bismut for his papers [B2] and [B-L] which enable us to simplify the previous version [Y1].

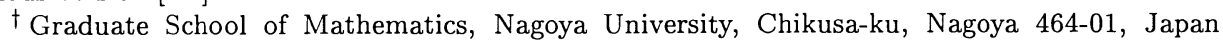
(yosikawa@math.nagoya-u.ac.jp). 
We apply Main Theorem to study the asymptotic behavior of analytic torsion in the case of smoothing of IHS. In the sequel of this section, we assume that $(E, h)$ is the trivial line bundle with the trivial metric. One of the consequence of Main Theorem is that principal term of the asymptotic behavior of analytic torsion is determined by the total Milnor number of the singular fiber and determinant of period integrals. (For a related result, see [Fa].) To be precise, let us fix notations.

Let $H$ be a relatively very ample line bundle over $X$ and $g_{X}$ the Kähler metric of $X$ whose Kähler class coincides with $c_{1}(H)$. Let $\tau\left(X_{t}\right)$ be the Ray-Singer analytic torsion of the smooth fiber $\left(X_{t},\left.g\right|_{X_{t}}\right)$. Fix a base point $t_{0} \neq 0$. Let $H_{n}\left(X_{t_{0}} ; \mathbb{Z}\right)_{f r}$ be the free part of $H_{n}\left(X_{t_{0}} ; \mathbb{Z}\right)$ and fix its integral basis, say $\left\{a_{1}, \cdots, a_{l}\right\}$ where $l=\operatorname{dim} H^{n}\left(X_{t_{0}}, \mathbb{R}\right)$. Let $\langle\cdot, \cdot\rangle$ be the intersection form on $H_{n}\left(X_{t_{0}}, \mathbb{Z}\right)$ and $\Lambda:=$ $\left(\left\langle a_{i}, a_{j}\right\rangle\right)_{1 \leq i, j \leq l}$ the intersection matrix relative to above basis. For any $t \in S^{*}$, fix an integral basis $\left\{a_{1}(t), \cdots, a_{l}(t)\right\}$ of $H_{n}\left(X_{t} ; \mathbb{Z}\right)_{f r}$ whose intersection matrix is $\Lambda$. Let $\omega_{X / S}:=\operatorname{det}(T X / S)^{*}$ be the relative canonical bundle of $(\pi, X, S)$. Fix a $\mathcal{O}_{S}$-basis of $\pi_{*} \omega_{X / S}$, say $\left\{\omega_{1}, \cdots, \omega_{m}\right\}$ where $m=\operatorname{dim} H^{n}\left(X_{t}, \mathcal{O}_{X_{t_{0}}}\right)$. Let $\Omega(t):=$ $\left(\int_{a_{i}(t)} \omega_{j}(t)\right)_{1 \leq i \leq l, 1 \leq j \leq m}$ be the period matrix relative to $\left\{a_{1}, \cdots, a_{l}\right\}$ and $\left\{\omega_{1}, \cdots\right.$, $\left.\omega_{m}\right\}$.

Theorem 0.1. There exist $r_{1}, \cdots, r_{k} \in \mathbb{Q}$ such that as $t \rightarrow 0$,

$$
\tau\left(X_{t}\right) \sim \sum_{r=r_{1}, \cdots, r_{k}} \sum_{i, j \geq 0} \sum_{k \geq-h^{n, 0} \cdot n} a_{r i j k}|t|^{r} t^{i} \bar{t}^{j}(\log |t|)^{k}
$$

where $h^{n, 0}=h^{n, 0}\left(X_{t}\right)(t \neq 0)$. Furthermore, there exists a constant $A$ such that

$$
\lim _{t \rightarrow 0} \frac{\tau\left(X_{t}\right)}{|t|^{2 \frac{(-1)^{n}}{(n+2) !} \mu\left(\operatorname{Sing} X_{0}\right)} \operatorname{det}\left({ }^{t} \Omega(t) \Lambda \bar{\Omega}(t)\right)^{(-1)^{n}}}=\exp (A) .
$$

As an application of Main Theorem, we show in [Y2] that analytic torsion of smooth theta divisor is represented by the Siegel modular form characterizing the Andreotti-Mayer locus.

1. Determinant bundles and Quillen metrics. In this section, we recall some basic properties of Quillen metrics in the simplest setting. For the general treatment, see $[\mathrm{S}]$ and $[\mathrm{F}]$.

Let $\pi: X \rightarrow S$ be a proper smooth morphism of Kähler manifolds. Let $(E, h)$ be a holomorphic Hermitian vector bundle over $X$. The determinant bundle $\lambda(E)$ is defined by the following formula:

$$
\lambda(E):=\bigotimes_{q \geq 0}\left(\operatorname{det} R^{q} \pi_{*} \mathcal{O}_{X}(E)\right)^{(-1)^{q}} .
$$

Let $g_{X / S}$ be a Kähler metric on the relative tangent bundle $T X / S:=\operatorname{ker} \pi_{*}$. Namely $\left.g_{X / S}\right|_{X_{t}}$ is Kähler for any fiber $X_{t}:=\pi^{-1}(t)$. Via the Hodge theory, identify the fiber $\lambda_{X_{t}}\left(E_{t}\right)=\lambda(E)_{t}$ with the determinant of harmonic forms:

$$
\lambda(E)_{t}=\bigotimes_{q \geq 0}\left(\bigwedge^{\max } H^{q}\left(X_{t}, E_{t}\right)\right)^{(-1)^{q}} \cong \bigotimes_{q \geq 0}\left(\bigwedge^{\max } \mathcal{H}^{0, q}\left(X_{t}, E_{t}\right)\right)^{(-1)^{q}}
$$

where $\mathcal{H}^{0, q}\left(X_{t}, E_{t}\right)$ stands for the harmonic $(0, q)$-forms with coefficients in $E$. Since $\mathcal{H}^{0, q}\left(X_{t}, E_{t}\right)$ carries the natural Hermitian structure by the integration of harmonic forms, so does $\lambda(E)_{t}$ via the identification (1.2). This metric is called the $L^{2}$-metric 
of $\lambda(E)$ relative to $g_{X / S}$ and $h$, and is denoted by $\|\cdot\|_{L^{2}}$. Let $\square_{t}^{0, q}$ be the $\bar{\partial}$-Laplacian acting on $E$-valued $(0, q)$-forms on $X_{t}$ and $\zeta_{t}^{0, q}(s)$ its zeta function. Then $\zeta_{t}^{0, q}(s)$ extends to a meromorphic function on the complex plane and is regular at $s=0$.

Definition 1.1. The Quillen metric of $\lambda(E)$ relative to $g_{X / S}$ and $h$ is defined by

$$
\|\cdot\|_{Q}^{2}(t):=\tau\left(X_{t}, E_{t}\right)\|\cdot\|_{L^{2}}^{2}(t)
$$

where $\tau\left(X_{t}, E_{t}\right)$ is the Ray-Singer analytic torsion:

$$
\tau\left(X_{t}, E_{t}\right):=\prod_{q \geq 0}\left(\operatorname{det} \square_{t}^{0, q}\right)^{(-1)^{q} q}, \quad \operatorname{det} \square_{t}^{0, q}:=\exp \left(-\left.\frac{d}{d s}\right|_{s=0} \zeta_{t}^{0, q}(s)\right) .
$$

It is known that $\|\cdot\|_{Q}$ is a smooth Hermitian metric on $\lambda(E)$ if the morphism is smooth. For smooth Kähler morphisms, curvature and anomaly of the Quillen metric is computed by Bismut-Gillet-Soulé ([B-G-S]).

THEOREM 1.1. Let $(\pi, X, S)$ be a locally Kähler smooth morphism of complex manifolds. Let $g_{X / S}$ be a Kähler metric of $T X / S$. Let $T d\left(T X / S, g_{X / S}\right)$ be the Todd form of $\left(T X / S, g_{X / S}\right)$ and $c h(E, h)$ the Chern character of $(E, h)$. Then,

$$
c_{1}\left(\lambda\left(\mathcal{O}_{X}\right),\|\cdot\|_{Q}\right)=\pi_{*}\left(T d\left(T X / S, g_{X / S}\right) \operatorname{ch}(E, h)\right)^{(1,1)}
$$

where $\alpha^{(p, p)}$ stands for the $(p, p)$-part of the form $\alpha$.

THEOREM 1.2. Let $g_{X / S}$ and $g_{X / S}^{\prime}$ be Kähler metrics of $T X / S$. Let $\|\cdot\|_{Q}$ and $\|\cdot\|_{Q}^{\prime}$ be the Quillen metrics of $\lambda(E)$ relative to $g_{X / S}$ and $g_{X / S}^{\prime}$ respectively. Then,

$$
\log \left(\frac{\|\cdot\|_{Q}^{\prime}}{\|\cdot\|_{Q}}\right)^{2}=\pi_{*}\left(\widetilde{T d}\left(T X / S ; g_{X / S}, g_{X / S}^{\prime}\right) \operatorname{ch}(E, h)\right)^{(0,0)}
$$

where $\widetilde{T d}\left(T X / S ; g_{X / S}, g_{X / S}^{\prime}\right)$ is the Bott-Chern class of $T X / S$ relative to the Todd form, $g_{X / S}$ and $g_{X / S}^{\prime}$.

THEOREM 1.3. Let $h$ and $h^{\prime}$ be Hermitian metrics of $E$. Let $\|\cdot\|_{Q}$ and $\|\cdot\|_{Q}^{\prime}$ be the Quillen metrics of $\lambda(E)$ relative to $h$ and $h^{\prime}$. Then,

$$
\log \left(\frac{\|\cdot\|_{Q}^{\prime}}{\|\cdot\|_{Q}}\right)^{2}=\pi_{*}\left(T d\left(T X / S, g_{X / S}\right) \widetilde{\operatorname{ch}}(E, h)\right)^{(0,0)}
$$

where $\widetilde{\operatorname{ch}}\left(E ; h, h^{\prime}\right)$ is the Bott-Chern class of $E$ relative to the Chern character, $h$ and $h^{\prime}$.

We also need a theorem of Bismut and Lebeau ([B-L]) which describes the Quillen norm of canonical section in terms of some secondary classes. (For the general cases, see $[B-L]$.)

THEOREM 1.4. Let $X$ be a compact Kähler manifold and $\left(Y, g_{Y}=\left.g_{X}\right|_{Y}\right)$ its smooth hypersurface with induced metric. Let $L=[Y]$ be the line bundle defined by $Y$ and $s_{Y}$ its canonical section, i.e., $\left(s_{Y}\right)_{0}=[Y]$. Let $h_{L}=\|\cdot\|_{L}^{2}$ be a Hermitian metric of $L$ and $g_{N_{Y / X}}$ a Hermitian metric of $N_{Y / X}$ such that, on $Y,\left\|d s_{Y}\right\|_{N_{Y / X}^{*} \otimes L_{Y}}^{2} \equiv 1$ where $L_{Y}:=\left.L\right|_{Y}$ and $d s_{Y} \in H^{0}\left(Y, N_{Y / X}^{*} \otimes L\right)$. Let $\lambda_{X}\left(E \otimes L^{-1}\right), \lambda_{X}(E)$ and $\lambda_{Y}\left(E_{Y}\right)$ be the determinant of cohomologies equipped with the Quillen metrics relative to $g_{X}, g_{Y}$ and $h_{E \otimes L^{-1}}, h_{E}, h_{E_{Y}}$. Let $\sigma$ be the canonical element of $\lambda:=\lambda_{Y}\left(E_{Y}\right) \otimes \lambda_{X}(E)^{-1} \otimes$ 
$\lambda_{X}\left(E \otimes L^{-1}\right)$. Then,

$$
\begin{aligned}
\log \|\sigma\|_{\lambda, Q}^{2}= & -\int_{X} \operatorname{Td}\left(T X, g_{X}\right) T d^{-1}\left(L, h_{L}\right) \operatorname{ch}(E, h) \log \|s\|_{L}^{2} \\
& +\int_{Y} T d^{-1}\left(N_{Y / X}, g_{N_{Y / X}}\right) \widetilde{T d}(\overline{\mathcal{E}}) \operatorname{ch}\left(E_{Y}\right) \\
& -\int_{X} T d(T X) R(T X) \operatorname{ch}(E)+\int_{Y} T d(T Y) R(T Y) \operatorname{ch}\left(E_{Y}\right)
\end{aligned}
$$

where $R$ is the Gillet-Soule genus and $\widetilde{T d}(\overline{\mathcal{E}})$ is the Bott-Chern class relative to the Todd genus and $\overline{\mathcal{E}}=\left\{\mathcal{E},\left(g_{Y}, g_{\left.T X\right|_{Y}}, g_{N_{Y / X}}\right)\right\}:\left.0 \rightarrow T Y \rightarrow T X\right|_{Y} \rightarrow N_{Y / X} \rightarrow 0$.

Recall the definition of $\widetilde{T d}(\overline{\mathcal{E}})$. Let $\mathcal{E}: 0 \rightarrow E^{\prime} \stackrel{i}{\rightarrow} E \stackrel{v}{\rightarrow} E^{\prime \prime} \rightarrow 0$ be an exact sequence of vector bundles over a complex manifold $M$ with Hermitian metrics $h_{E^{\prime}}, h_{E}, h_{E^{\prime \prime}}$. Define a vector bundle over $M \times \mathbb{P}^{1}$ by

$$
\tilde{E}=\left\{\left(e, e^{\prime \prime}\right) \in E \oplus E^{\prime \prime}(-1) ; v(e)+\sigma_{\infty} \otimes e^{\prime \prime}=0\right\}
$$

where $E=: p r_{1}^{*} E, E^{\prime \prime}(-1):=p r_{1}^{*} E^{\prime \prime} \otimes \mathcal{O}_{\mathbb{P}^{1}}(-1), \sigma_{0}$ and $\sigma_{\infty}$ the canonical section of $\mathcal{O}_{\mathbb{P}^{1}}(1)$ such that $\left(\sigma_{0}\right)_{0}=0$ and $\left(\sigma_{\infty}\right)_{0}=\infty$. In the homogeneous coordinate, $\sigma_{0}=z_{0}$ and $\sigma_{\infty}=z_{1}$ where $z=z_{0} / z_{1}$ is the inhomogeneous coordinate of $\mathbb{P}^{1}$. Let

$$
h_{\tilde{E}}^{\prime}\left(\left(e, e^{\prime \prime}\right),\left(e, e^{\prime \prime}\right)\right):=h_{E}(e, e)+h_{E^{\prime \prime}}\left(\sigma_{0} \otimes e^{\prime \prime}, \sigma_{0} \otimes e^{\prime \prime}\right)
$$

be a Hermitian metric of $\tilde{E}$. Consider the following exact sequence of vector bundles on $M \times \mathbb{P}^{1}: 0 \rightarrow E^{\prime} \stackrel{i \oplus 0}{\rightarrow} \tilde{E} \stackrel{p_{2}}{\rightarrow} E^{\prime \prime}(-1) \rightarrow 0$ where $p_{2}: \tilde{E} \ni\left(e, e^{\prime \prime}\right) \rightarrow e^{\prime \prime} \in E^{\prime \prime}(-1)$. Then,

$$
\widetilde{T d}(\overline{\mathcal{E}})=\int_{\mathbb{P}^{1}} \log |z|^{2} \cdot T d\left(\tilde{E}, h_{\tilde{E}}^{\prime}\right) .
$$

2. Families of hypersurfaces and relative Todd form. Let $S$ be a complex manifold and $D=\{z \in \mathbb{C} ;|z|<1\}$ be the unit disc. Through this section, $S$ is not necessary the unit disc. Put $U:=D^{n+1} \times S$. Let $F(z, s) \in \mathcal{O}(U)$ be a holomorphic function such that $F(\cdot, s)$ is not a constant function for any $s \in S$ where $z=\left(z_{0}, \cdots, z_{n}\right) \in D^{n+1}$. Set

$$
\begin{aligned}
& X:=\{(z, s) \in U ; F(z, s)=0\} \subset U, \\
& p: U \ni(z, s) \rightarrow s \in S, \quad \pi:=\left.p\right|_{X}: X \rightarrow S, \\
& \Sigma:=\left\{(z, s) \in U ; d_{\mathbb{C}^{n}} F:=\sum_{i=0}^{n} \frac{\partial F}{\partial z_{i}} d z^{i}=0\right\} .
\end{aligned}
$$

Let $T \mathbb{C}^{n+1}:=\left.\operatorname{ker} p_{*}\right|_{X}$ and $T X / S:=\operatorname{ker} \pi_{*}$ be the vector bundles associated to $p$ and $\pi$ respectively defined on $U-\Sigma$. Put $N_{X / \mathbb{C}^{n+1}}:=\operatorname{ker} p_{*} / \operatorname{ker} \pi_{*}$ and consider the following exact sequence:

$$
0 \longrightarrow T X / S \longrightarrow T \mathbb{C}^{n+1} \longrightarrow N_{X / \mathbb{C}^{n+1}} \longrightarrow 0 \text {. }
$$

Let $g_{\mathbb{C}^{n+1}}=\sum_{i=0}^{n}\left|d z_{i}\right|^{2}$ be the Euclidean metric of $T \mathbb{C}^{n+1}$ which gives the smooth orthogonal splitting $T \mathbb{C}^{n+1}=T X / S \oplus(T X / S)^{\perp}$. Put $g_{X / S}=\left.g_{\mathbb{C}^{n+1}}\right|_{T X / S}$ for the induced metric of $T X / S . \quad N_{X / \mathbb{C}^{n+1}}$ is equipped with the Hermitian metric $g_{N}=$ $g_{(T X / S)^{\perp}}$ by the $C^{\infty}$-identification $N_{X / \mathbb{C}^{n+1}} \cong(T X / S)^{\perp}$. Let $R_{X / S}$ be the curvature form of $\left(T X / S, g_{X / S}\right)$ defined on $U-\Sigma$. 
Proposition 2.1. On $U-\Sigma$, it holds that $\left[\operatorname{Td}\left(R_{X / S}\right)\right]^{(n+1, n+1)} \equiv 0$.

For the proof, we need the followings.

Lemma 2.1. Let $V$ be a vector space over $\mathbb{C}$. For any $A=\left(a_{0}, \cdots, a_{n}\right), B=$ $\left(b_{0}, \cdots, b_{n}\right) \in V \otimes \mathbb{C}^{n+1}\left(a_{i}, b_{j} \in V\right)$ and indeterminate $x$,

$$
\operatorname{det}\left(I+x^{t} B \wedge A\right)=\left(1+x A \wedge^{t} B\right)^{-1} \text {. }
$$

Here ${ }^{t} B \wedge A=\left(b_{i} \wedge a_{j}\right) \in M\left(n, \wedge^{*} V\right)$ and $A \wedge^{t} B=\sum a_{i} \wedge b_{j} \in \wedge^{*} V$.

Proof. Since $\left(A \wedge{ }^{t} B\right)^{k}=-\operatorname{Tr}\left({ }^{t} B \wedge A\right)^{k},\left(A \wedge{ }^{t} B\right)^{l}=0(l>\operatorname{dim} V)$, and

$$
\operatorname{det}(I-t X)=\exp \left(-\sum_{i=1}^{\infty} \frac{\operatorname{Tr}\left(X^{i}\right)}{i} t^{i}\right)
$$

for any square matrix $X$, we get the formula by putting $t=-x$ and $X={ }^{t} B \wedge A$.

LEMmA 2.2. For any $F(x)=1+\sum_{i \geq 1} a_{i} x^{i} \in \mathbb{C}[[x]]$ and $A, B \in V \otimes \mathbb{C}^{n}$,

$$
\operatorname{det} F\left({ }^{t} B \wedge A\right)=F\left(A \wedge{ }^{t} B\right)^{-1} \text {. }
$$

Proof. Put $F\left({ }^{t} B \wedge A\right)=I+y^{t} B \wedge A$ and $y=\sum_{i \geq 1} a_{i}\left(A \wedge{ }^{t} B\right)^{i-1}$. By Lemma 2.1, $\operatorname{det} F\left({ }^{t} B \wedge A\right)=\left(1+y A \wedge^{t} B\right)^{-1}=F\left(A \wedge{ }^{t} B\right)^{-1}$.

Proof of Proposition 2.1. Let $R_{N}$ be the curvature form of $\left(N_{X / \mathbb{C}^{n+1}}, g_{N}\right)$. Let $A \in A_{U}^{1,0}\left(\operatorname{Hom}\left(T X / S,(T X / S)^{\perp}\right)\right)$ be the second fundamental form of (2.3). By the Gauss-Codazzi equation ([Ko], I (6.1)), the curvature form of $\left(T \mathbb{C}^{n+1}, g_{\mathbb{C}^{n+1}}\right)$ is represented by

$$
R_{T \mathbb{C}^{n+1}}=\left(\begin{array}{cc}
R_{X / S}-A^{*} \wedge A & -D^{\prime} A^{*} \\
D^{\prime \prime} A & R_{N}-A \wedge A^{*}
\end{array}\right)
$$

Since $\left(T \mathbb{C}^{n+1}, g_{\mathbb{C}^{n+1}}\right)$ is a flat vector bundle, we find $R_{T \mathbb{C}^{n+1}} \equiv 0$ which implies

$$
R_{X / S}=A^{*} \wedge A, \quad R_{N}=A \wedge A^{*}, \quad D^{\prime \prime} A=D^{\prime} A^{*}=0 .
$$

Applying Lemma 2.2 for $R_{X / S}$ and $F(x)=T d(x)=x /\left(1-e^{-x}\right)$, we get

$$
\operatorname{Td}\left(R_{X / S}\right)=\operatorname{Td}\left(R_{N}\right)^{-1}
$$

In particular,

$$
\left[T d\left(R_{X / S}\right)\right]^{(n+1, n+1)}=(-1)^{n+1} \frac{1}{(n+2) !}\left(\frac{i}{2 \pi} R_{N}\right)^{n+1} .
$$

Consider the dual of (2.3):

$$
0 \longrightarrow N_{X / \mathbb{C}^{n+1}}^{*} \longrightarrow \Omega_{\mathbb{C}^{n+1}}^{1} \longrightarrow \Omega_{X / S}^{1} \longrightarrow 0
$$

As $N_{X / \mathbb{C}^{n+1}}^{*}$ is generated by $d_{\mathbb{C}^{n+1}} F=\sum_{i=0}^{n} \frac{\partial F}{\partial z_{i}}(z, s) d z^{i}, R_{N}$ is represented by

$$
R_{N}=-R_{N_{X / \mathbb{C}^{n+1}}}^{*}=-\bar{\partial} \partial \log \left\|d_{\mathbb{C}^{n+1}} F\right\|^{2}, \quad\left\|d_{\mathbb{C}^{n+1}} F\right\|^{2}=\sum_{i=0}^{n}\left|\frac{\partial F}{\partial z_{i}}(z, s)\right|^{2} .
$$

Let $\nu: U-\Sigma \rightarrow \mathbb{P}^{n}$ be the Gauss map:

$$
\nu: U-\Sigma \ni(z, s) \rightarrow\left[\frac{\partial F}{\partial z_{0}}(z, s): \cdots: \frac{\partial F}{\partial z_{n}}(z, s)\right] \in \mathbb{P}^{n}
$$


By (2.11), we find

$$
\frac{i}{2 \pi} R_{N}=\nu^{*} \omega_{\mathbb{P}^{n}}, \quad \omega_{\mathbb{P}^{n}}=\frac{i}{2 \pi} \partial \bar{\partial} \log \|z\|^{2}
$$

which combined with (2.9) yields

$$
\left[T d\left(R_{X / S}\right)\right]^{(n+1, n+1)}=\frac{(-1)^{n+1}}{(n+2) !} \nu^{*} \omega_{\mathbb{P}^{n}}^{n+1}=0 .
$$

3. Estimates of anomaly. Let $(\pi, X, S)$ be the smoothing of IHS as before. Let $g_{X}$ and $g_{X}^{\prime}$ be Kähler metrics of $X$. The induced metric on $T X / S$ are denoted by $g_{X / S}$ and $g_{X / S}^{\prime}$. Let $E$ be a holomorphic vector bundle over $X$ and $h, h^{\prime}$ its Hermitian metrics. We prove the following theorem in this section.

Theorem 3.1. There exists $\alpha>0, r_{1}, \cdots, r_{a} \in \mathbb{Q}_{\geq 0}$ and $p>1$ depending only on Sing $X_{0}$ such that

(1) $\pi_{*}\left(\widetilde{T d}\left(T X / S ; g_{X / S}, g_{X / S}^{\prime}\right) \operatorname{ch}(E, h)\right)^{(0,0)}$ lies in $C^{\alpha}(S)$ and admits the following asymptotic expansion as $t \rightarrow 0$ :

$$
\pi_{*}\left(\widetilde{T d}\left(T X / S ; g_{X / S}, g_{X / S}^{\prime}\right) \operatorname{ch}(E, h)\right)^{(0,0)} \sim \sum_{r} \sum_{i, j \geq 0} \sum_{0 \leq k \leq n} a_{r i j k}|t|^{r} t^{i} \bar{t}^{j}(\log |t|)^{k}
$$

(2) $\pi_{*}\left(T d\left(T X / S, g_{X / S}\right) \operatorname{ch}(E, h)\right)^{(1,1)}$ lies in $L_{l o c}^{p}(S)$,

(3) $\pi_{*}\left(T d\left(T X / S, g_{X / S}\right) \widetilde{c h}\left(E ; h, h^{\prime}\right)\right)^{(0,0)}$ lies in $C^{\alpha}(S)$ and admits the following asymptotic expansion as $t \rightarrow 0$ :

$$
\pi_{*}\left(T d\left(T X / S, g_{X / S}\right) \widetilde{c h}\left(E ; h, h^{\prime}\right)\right)^{(0,0)} \sim \sum_{r} \sum_{i, j \geq 0} \sum_{0 \leq k \leq n} b_{r i j k}|t|^{r} t^{i} \bar{t}^{j}(\log |t|)^{k} .
$$

(4) $\pi_{*}\left(T d\left(T X / S, g_{X / S}\right) \widetilde{c h}(E, h)\right)^{(1,1)}$ lies in $L_{l o c}^{p}(S)$.

For the proof, we need the following theorem due to Barlet ([Ba]).

TheOREM 3.2. Let $X$ be a complex manifold of dimension $n+1$ and $K$ a compact subset. Let $\phi \in \Omega_{0}^{n, n}(K)$ be a smooth $(n, n)$-form supported in $K$. Let $f: X \rightarrow$ $S$ be a holomorphic function. Then, $f_{*}(\phi)$ is a Hölder continuous function on $S$. Furthermore, there exists $r_{1}, \cdots, r_{k} \in \mathbb{Q} \cap[0,2)$ such that, as $t \rightarrow 0$,

$$
f_{*}(\phi)(t) \sim \sum_{r=r_{1}, \cdots, r_{k}} \sum_{i, j \geq 0} \sum_{0 \leq k \leq n} a_{r i j k}|t|^{r} t^{i} \bar{t}^{j}(\log |t|)^{k} .
$$

Proof of Theorem 3.1. Since the proof of (3) and (4) is similar to that of (1) and (2), we only prove the former. Furthermore, we assume $E$ is trivial and $h \equiv 1$ for simplicity because the problem is of local nature and the proof of the case $h \not \equiv 1$ is similar. Let $p: \mathbb{P}^{\vee}(T X) \rightarrow X$ be the projective bundle whose fiber at $x$, say $\mathbb{P}^{\vee}(T X)_{x}=\mathbb{P}^{\vee}\left(T_{x} X\right)$, is the set of all hyperplanes in $T_{x} X$. Let $F_{X}$ be the universal vector bundle over $\mathbb{P}^{\vee}(T X)$. Let $H_{x}$ be a hyperplane in $T_{x} X$ and $\left[H_{x}\right]$ the point in $\mathbb{P}^{\vee}\left(T_{x} X\right)$ corresponding to $H_{x}$. Then, $\left(F_{X}\right)_{\left(x,\left[H_{x}\right]\right)}=H_{x}$. By the construction, we obtain the following exact sequence of vector bundles over $\mathbb{P}^{\vee}(T X)$ :

$$
0 \longrightarrow F_{X} \longrightarrow p^{*} T X \longrightarrow N_{X} \longrightarrow 0
$$

where $N_{X}=\mathcal{O}_{\mathbb{P}^{\vee}(T X)}(1)$. Define Hermitian metrics on $F_{X}$ by $g_{F_{X}}:=\left.p^{*} g_{X}\right|_{F_{X}}$ and $g_{F_{X}}^{\prime}:=\left.p^{*} g_{X}^{\prime}\right|_{F_{X}}$. Let $\nu: T X / S \rightarrow F_{X}$ be the Gauss map:

$$
\nu: T_{x} X / S \ni(x, v) \rightarrow\left(x,\left[T_{x} X / S\right], v\right) \in F_{X,\left(x,\left[T_{x} X / S\right]\right)}
$$


The underlying map from $X$ to $\mathbb{P}^{\vee}(T X)$ is also denoted by $\nu$. Then, $\nu: X \rightarrow \mathbb{P}^{\vee}(T X)$ is a rational map which is holomorphic on $X-\operatorname{Sing} X_{0}$. From the universal property of $F_{X}$, it follows that $\left(T X / S, g_{X / S}\right)=\nu^{*}\left(F_{X}, g_{F_{X}}\right)$. In particular, we get

$T d\left(T X / S, g_{X / S}\right)=\nu^{*} T d\left(F_{X}, g_{F_{X}}\right), \quad \widetilde{T d}\left(T X / S ; g_{X / S}, g_{X / S}^{\prime}\right)=\nu^{*} \widetilde{T d}\left(F_{X} ; g_{F_{X}}, g_{F_{X}}^{\prime}\right)$.

Due to Hironaka, there exists a proper modification $f: Y \rightarrow X$ such that

(1) $f: Y-f^{-1}\left(\operatorname{Sing} X_{0}\right) \rightarrow X-\operatorname{Sing} X_{0}$ is an isomorphism,

(2) $(\pi \circ f)^{-1}(0)=\sum_{i} k_{i} D_{i}\left(k_{i}>0\right)$ is a divisor with simple normal crossing,

(3) There exists a holomorphic map $\phi: Y \rightarrow \mathbb{P}^{\vee}(T X)$ such that $\phi=\nu \circ f$ on $Y-$ $f^{-1}\left(\right.$ Sing $\left.X_{0}\right)$.

(1) Put $q:=\pi \circ f: Y \rightarrow S$. It follows from (3.3) that

$$
\pi_{*}\left(\widetilde{T d}\left(T X / S ; g_{X / S}, g_{X / S}^{\prime}\right)\right)^{(0,0)}=q_{*}\left(\phi^{*} \widetilde{T d}\left(F_{X} ; g_{F_{X}}, g_{F_{X}}^{\prime}\right)\right)^{(0,0)} .
$$

Since $\phi^{*} \widetilde{T d}\left(F_{X} ; g_{F_{X}}, g_{F_{X}}^{\prime}\right)$ is a smooth $(n, n)$-form on $Y$, we get the assertion by Theorem 3.2 .

(2) By the assumption, for any $w \in Y$, there exist local coordinates $\left(U_{w},\left(w_{0}, \cdots, w_{n}\right)\right)$ such that $q(w)=w_{0}^{k_{0}} \cdots w_{l}^{k_{l}}\left(k_{i} \geq 1, l \leq n\right)$. Set

$$
\tau:=\frac{1}{l} \sum_{i=1}^{l} \frac{1}{k_{i}}(-1)^{i-1} w_{i} d w_{0} \wedge \cdots \wedge d w_{i-1} \wedge d w_{i+1} \wedge \cdots \wedge d w_{n} .
$$

Let $\rho_{U_{w}}$ be a smooth function supported in $U_{w}$. Since $\phi^{*} T d\left(T X / S, g_{X / S}\right)$ is a smooth $(n+1, n+1)$-form on $U_{w}$, there exists $h \in C_{0}^{\infty}\left(U_{w}\right)$ such that

$$
\rho_{U_{w}} \phi^{*} T d\left(T X / S, g_{X / S}\right)=h d w_{0} \wedge d \bar{w}_{0} \wedge \cdots \wedge d w_{n} \wedge d \bar{w}_{n} .
$$

Since $q^{*}(d t / t) \wedge \tau=d w_{0} \wedge \cdots \wedge d w_{n}$, we get

$$
\begin{aligned}
q_{*}\left(\rho_{U_{w}} \phi^{*} T d\left(T X / S, g_{X / S}\right)\right)^{(1,1)}(t) & =q_{*}\left(h d w_{0} \wedge d \bar{w}_{0} \wedge \cdots \wedge d w_{n} \wedge d \bar{w}_{n}\right)(t) \\
& =\frac{d t \wedge d \bar{t}}{|t|^{2}} \int_{q^{-1}(t) \cap U_{w}} h \tau \wedge \bar{\tau}
\end{aligned}
$$

By Theorem $3.2, \pi_{*}\left(\rho_{U_{w}} T d\left(T X / S, g_{X / S}\right)\right)^{(1,1)}$ has the asymptotic expansion

$$
q_{*}\left(\rho_{U_{w}} \phi^{*} T d\left(T X / S, g_{X / S}\right)\right)^{(1,1)}(t) \sim \frac{d t \wedge d \bar{t}}{|t|^{2}} \sum_{r} \sum_{i, j \geq 0} \sum_{0 \leq k \leq n} c_{r i j k}|t|^{r} t^{i} \bar{t}^{j}(\log |t|)^{k} .
$$

Put $S_{\epsilon}=\{t \in S ;|t|<\epsilon\} \Subset S$. Since

$$
\left.\left|\int_{S_{\epsilon}} q_{*}\left(\rho_{U_{w}} \phi^{*} T d\left(T X / S, g_{X / S}\right)\right)^{(1,1)}\right|=\mid \int_{q^{-1}\left(S_{\epsilon}\right)} \rho_{U_{w}} \phi^{*} T d\left(T X / S, g_{X / S}\right)\right)^{(1,1)} \mid<\infty,
$$

$c_{r i j k} \neq 0$ if and only if $r+i+j>0$. In particular, there exists $p>1$ such that $\pi^{*}\left(\operatorname{Td}\left(T X / S, g_{X / S}\right)\right)^{(1,1)} \in L_{l o c}^{p}(S)$. This proves (2).

4. An analytic characterization of Milnor number. Let $D$ be the unit disc in $\mathbb{C}$. We prove the following theorem in this section.

THEOREM 4.1. Let $f(z)$ be a holomorphic function defined on the polydisc $D^{n+1}$ centered at $0 \in \mathbb{C}^{n+1}$ such that the origin is an isolated critical point of $f$. Set 
$Y_{t}:=D^{n+1} \cap f^{-1}(t)$ for the fiber of $f$. Let $\mu(f)$ be the Milnor number of $\left(Y_{0}, 0\right)$.

Then,

$$
\int_{Y_{t}}\left(\frac{i}{2 \pi} \partial \bar{\partial} \log \|d f\|^{2}\right)^{n} \log \|d f\|^{2}=\mu(f) \log |t|^{2}+O(1) \quad(t \rightarrow 0) .
$$

Proof. At first, let us consider the case that $f(z)$ has non-degenerate singularity at $z=0$. There exist local coordinates $\left(U,\left(w_{0}, \cdots, w_{n}\right)\right)$ around the critical point of $f$ such that $f(w)=w_{0}^{2}+\cdots+w_{n}^{2}$. Let $g_{1}:=\sum_{i=0}^{n}\left|d w_{i}\right|^{2}$ be a flat Kähler metric on $U$. Various norms relative to $g_{1}$ is denoted by $\|\cdot\|_{1}$. By Stokes's theorem,

$$
\begin{aligned}
I(t):= & \int_{Y_{t}}\left(\frac{i}{2 \pi} \partial \bar{\partial} \log \|d f\|^{2}\right)^{n} \log \|d f\|^{2} \\
= & \int_{Y_{t}}\left(\frac{i}{2 \pi} \partial \bar{\partial} \log \|d f\|_{1}^{2}\right)^{n} \log \|d f\|_{1}^{2} \\
& +\int_{Y_{t}} \log \frac{\|d f\|^{2}}{\|d f\|_{1}^{2}} \cdot \sum_{i+j=n}\left(\frac{i}{2 \pi} \partial \bar{\partial} \log \|d f\|^{2}\right)^{i}\left(\frac{i}{2 \pi} \partial \bar{\partial} \log \|d f\|_{1}^{2}\right)^{j}+O(1)
\end{aligned}
$$

where the $O(1)$-term comes from the integral along the boundary. Let $q: V \rightarrow D^{g}$ be the blowing-up at 0 . Then, $q^{*} \log \frac{\|d f\|^{2}}{\|d f\|_{1}^{2}}$ extends to a smooth function on $V$. Therefore, the second integral of (4.1) is a continuous function by Theorem 3.2. Since $\|d f\|_{1}^{2}(w)=$ $\|w\|^{2}$, we get

$$
I(t)=\int_{w_{0}^{2}+\cdots+w_{n}^{2}=t}\left(\frac{i}{2 \pi} \partial \vec{\partial} \log \|w\|^{2}\right)^{n} \log \|w\|^{2}+O(1)=\log |t|^{2}+O(1) .
$$

This proves the case of non-degenerate critical point.

Consider the general case. Let $F(z, s)$ be a one parameter deformation of $f(z)$ defined on $D^{n+2}$ such that $F(z, 0)=f(z)$. Put $f_{s}(z):=F(z, s)$. Let $Y_{(t, s)}:=f_{s}^{-1}(t)$ be the fiber of the map $\pi:(z, s) \rightarrow\left(f_{s}(z), s\right)$. Let $\Delta$ be the divisor of $D^{n+2}$ over which the fiber of $\pi$ is singular. We may assume that $f_{s}(s \neq 0)$ has only finitely many non-degenerate critical points by choosing $F(x, s)$ as the Morsification of $f(z)$. Let $\rho$ be a cut-off function on $D^{n+2}$ such that $\rho \equiv 1$ on a neighborhood of 0 , say $W$, and $\rho \equiv 0$ on a neighborhood of $\partial D^{n+2}$. Put

$$
\phi(t, s):=\int_{Y_{(t, s)}} \rho \cdot\left(\frac{i}{2 \pi} \partial \bar{\partial} \log \|d F\|^{2}\right)^{n} \log \|d F\|^{2}, \quad \chi:=\frac{i}{2 \pi} \partial \bar{\partial} \phi .
$$

Then,

$$
\chi=\frac{i}{2 \pi} \int_{Y_{(t, s)}-W} \rho \cdot\left(\frac{i}{2 \pi} \partial \bar{\partial} \log \|d F\|^{2}\right)^{n+1}+\int_{Y_{(t, s)}} \partial \rho \wedge F_{1}+\bar{\partial} \rho \wedge F_{2}+\partial \bar{\partial} \rho \wedge F_{3}
$$

for some smooth forms $F_{1}, F_{2}, F_{3}$ because $\left(\partial \bar{\partial} \log \|d F\|^{2}\right)^{n+1} \equiv 0$ on $W$ (cf. (2.14)). Thus $\chi$ extends to a smooth real $(1,1)$-form over $D^{2}$. Since $\left.\phi\right|_{\gamma}=\log |t|^{2}+\psi(\psi \in$ $C^{\infty}(\gamma)$ ) for any holomorphic curve $\gamma$ which intersects transversally to $\Delta$ by (4.2), applying the argument of [B-B, $\S 10.2]$, it follows that

$$
\frac{i}{2 \pi} \partial \bar{\partial} \phi=\delta_{\Delta}-\chi
$$

in the sense of current over $D^{2}$. Since the multiplicity of $\Delta$ at 0 is $\mu(f)$ (cf. [Lo]), 
restricting (4.5) to the disc $D \times\{0\}$, we get

$$
\frac{i}{2 \pi} \partial \bar{\partial}\left(\left.\phi\right|_{D \times\{0\}}\right)=\text { mult }_{0} \Delta \cdot \delta_{0}-\left.\chi\right|_{D \times\{0\}}=\mu(f) \delta_{0}-\left.\chi\right|_{D \times\{0\}} .
$$

The assertion follows from (4.6).

5. An explicit formula for the Bott-Chern form. Let $X=D^{n+1}$ be the polydisc and $Y=\{z \in X ; \theta(z)=0\}$ its smooth hypersurface defined by $\theta \in \mathcal{O}(X)$ through this section. Consider the following exact sequence of vector bundles over $Y$ :

$$
\mathcal{E}:\left.0 \longrightarrow T Y \longrightarrow T X\right|_{Y} \longrightarrow N_{Y / X} \longrightarrow 0 .
$$

Let $g_{X}=\sum_{i=0}^{n}\left|d z_{i}\right|^{2}$ be the flat metric on $T X$ and $g_{Y}$ the induced metric on $T Y$. Let $g_{N}$ be a Hermitian metric of $N_{Y / X}$ induced by the $C^{\infty}$-identification $(T Y)^{\perp} \cong N_{Y / X}$. Let $g_{N}^{\prime}$ be another metric on $N$ such that $g_{N^{*}}^{\prime}(d \theta, d \theta) \equiv 1$ where $g_{N^{*}}^{\prime}:=\left(g_{N}^{\prime}\right)^{-1}$ and $d \theta$ the generator of $N^{*}$. Set $\overline{\mathcal{E}}:=\left(\mathcal{E},\left(g_{T Y}, g_{X}, g_{N}^{\prime}\right)\right)$. We prove the following theorem in this section.

THEOREM 5.1.

$$
\widetilde{T d}(\overline{\mathcal{E}})=\frac{1-T d\left(\nu^{*} c_{1}(H)\right)}{\nu^{*} c_{1}(H)} \log \|d \theta\|^{2}+\int_{0}^{1} \log \frac{1-s}{s} \frac{d}{d s} \frac{T d^{-1}\left(s \nu^{*} c_{1}(H)\right)}{\nu^{*} c_{1}(H)} d s .
$$

where $\nu: D^{n+1} \ni z \rightarrow\left(\frac{\partial \theta}{\partial z_{0}}(z): \cdots: \frac{\partial \theta}{\partial z_{n}}(z)\right) \in \mathbb{P}^{n}$ is the Gauss map relative to $\theta$.

Before proving Theorem 5.1, let us verify that $\overline{\mathcal{E}}$ satisfies Bismut's condition (A) (cf. [B1]). Put $L=[Y]$ for the line bundle on $X$ defined by the divisor $Y$ and $L_{Y}:=\left.L\right|_{Y}$ its restriction to $Y$. As $L$ has the canonical section $s$ such that $(s)_{0}=Y, s_{1}:=\theta^{-1} s$ is a nowhere vanishing section trivializing $L$. Let $h_{L}$ be the Hermitian metric of $L$ such that $h_{L}(s, s)=|\theta|^{2}$ and $h_{L_{Y}}$ its restriction to $L_{Y}$. Clearly $h_{L}\left(s_{1}, s_{1}\right) \equiv 1$ and the Chern form of $\left(L, h_{L}\right)$ vanishes on $X$. Now the normal bundle $N_{Y / X}$ is identified with $L_{Y}$ by the following map:

$$
\otimes d \theta: N_{Y / X} \ni\left[\frac{\partial}{\partial z_{i}}\right] \rightarrow \frac{\partial \theta}{\partial z_{i}} s_{1} \in L_{Y} .
$$

Let $p: N_{Y / X} \rightarrow Y$ be the projection. By the construction, the following is clear.

LEMMA 5.1. $\otimes d \theta:\left(p^{*} L_{Y}^{-1}, p^{*} h_{L_{Y}}^{-1}\right) \rightarrow\left(p^{*} N_{Y / X}, p^{*} g_{N^{*}}^{\prime}\right)$ is an isometry. Namely, $\left\|d \theta \otimes s_{1}\right\|_{N^{*} \otimes L_{Y}}^{\prime} \equiv 1$ where $\|\cdot\|_{N^{*} \otimes L_{Y}}^{\prime}:=g_{N^{*}}^{\prime} \otimes h_{L_{Y}}$.

In the sequel, identify $s_{1}=1$. We compute $\widetilde{T} d(\overline{\mathcal{E}})$ by using the formula (1.5). The Gauss map induces the following metric on $L_{Y}$ :

$$
\nu^{*} h_{H}\left(\frac{\partial \theta}{\partial z_{i}}, \frac{\partial \theta}{\partial z_{i}}\right)=\frac{\left|\frac{\partial \theta}{\partial z_{i}}\right|^{2}}{\|d \theta\|^{2}}, \quad\|d \theta\|^{2}:=\sum_{i=0}^{n}\left|\frac{\partial \theta}{\partial z_{i}}\right|^{2}
$$

where $h_{H}$ is the standard metric of $H=\mathcal{O}_{\mathbb{P}^{n}}(1)$. Its Chern form is given by

$$
c_{1}\left(L, \nu^{*} h_{H}\right)=\nu^{*} c_{1}(H), \quad c_{1}(H)=\frac{i}{2 \pi} \partial \bar{\partial} \log \|z\|^{2} .
$$

Define $\widetilde{T X}$ and $g_{\widetilde{T X}}^{\prime}$ in the same manner as (1.3) and (1.4).

Lemma 5.2. Put $u:=1 / z$ for the coordinate of $\mathbb{P}^{1}$ near $\infty$. Then,

$$
g_{\overparen{T X}}^{\prime}=g_{T Y} \oplus\left(1+\frac{\|d \theta\|^{2}}{|u|^{2}}\right) g_{N}
$$


Proof. By (1.4),

$$
g_{\overparen{T X}}^{\prime}=g_{Y} \oplus\left(g_{N}+\frac{1}{|u|^{2}} g_{N}^{\prime}\right)
$$

The assertion follows from the following:

$$
\frac{g_{N}^{\prime}}{g_{N}}=\frac{\|d \theta\|^{2}}{\|d \theta\|_{N^{*} \otimes L_{Y}}^{2}}=\|d \theta\|^{2} .
$$

Consider the following exact sequence of Hermitian vector bundles over $\mathbb{P}^{n}$ :

$$
0 \longrightarrow E \longrightarrow \mathbb{C}^{n+1} \longrightarrow H=\mathcal{O}_{\mathbb{P}^{n}}(1) \longrightarrow 0
$$

where $E$ is the universal bundle over $\mathbb{P}^{n}$, i.e., $E_{u}=\left\{z \in \mathbb{C}^{n+1} ; \sum_{i=0}^{n} u_{i} z_{i}=0\right\}$ for $u \in \mathbb{P}^{n}$. $\mathbb{C}^{n+1}$ is equipped with the standard Euclidean metric and $E$ and $H$ are equipped with the induced metric from (5.7). Then, the following is clear by the definition:

$$
\left(T Y, g_{T Y}\right)=\nu^{*}\left(E, h_{E}\right), \quad\left(N_{Y / X}, h_{N}\right)=\nu^{*}\left(H, h_{H}\right),
$$

which combined with Lemma 5.2 yields the following lemma.

Lemma 5.3. Put $\phi^{2}:=\|d \theta\|^{2}$. Then,

$$
g_{\overparen{T X}}^{\prime}=\nu^{*} h_{E} \oplus\left(1+\frac{\phi^{2}}{|u|^{2}}\right) \nu^{*} h_{H}
$$

Put $a(u, z):=1+|u|^{-2} \phi^{2}(z)$. Let $A$ be the second fundamental form of the exact sequence (5.7). As $\left(\mathbb{C}^{n+1}, h_{\mathbb{C}^{n+1}}\right)$ is flat, we have the following formula by the Gauss-Codazzi equation.

LEMMA 5.4.

$$
R_{\widetilde{T X}}=\left(\begin{array}{cc}
\left(1-\frac{1}{a}\right) \nu^{*} R_{E} & \partial \log a \wedge \nu^{*} A^{*} \\
-\frac{1}{a} \bar{\partial} \log a \wedge \nu^{*} A & \left(1-\frac{1}{a}\right) \nu^{*} R_{H}+\bar{\partial} \partial \log a
\end{array}\right)
$$

where $R_{\widetilde{T X}}, R_{E}$ and $R_{H}$ are curvatures of $\left(\widetilde{T X}, h_{\widetilde{T X}}^{\prime}\right),\left(E, h_{E}\right)$ and $\left(H, h_{H}\right)$.

Set

$$
v:=\phi u, \quad B:=\nu^{*} A, \quad x:=\partial \log \frac{\phi}{|v|^{2}}, \quad y:=\frac{|v|^{2}}{1+|v|^{2}} \bar{\partial} \log \frac{|v|^{2}}{\phi}, \quad \omega:=\bar{\partial} \partial \phi .
$$

Then, similar to (2.7), we get $B \wedge B^{*}+\omega=\nu^{*} R_{H}+\omega=0$. By Lemma 5.4, we have the following formula.

Proposition 5.1. Put $T:=\left(\begin{array}{cc}B^{*} \wedge B & x \wedge B^{*} \\ y \wedge B & x y\end{array}\right)$. Then,

$$
R:=R_{\widetilde{T X}}=\frac{1}{1+|v|^{2}} \cdot T \text {. }
$$

Lemma 5.5. Set $T_{\epsilon}=\left(\begin{array}{cc}B^{*} \wedge B & \epsilon x \wedge B^{*} \\ \epsilon y \wedge B & \epsilon^{2} x y\end{array}\right)=\left(\begin{array}{c}B^{*} \\ \epsilon y\end{array}\right)\left(\begin{array}{ll}B & -\epsilon x\end{array}\right)$. Then,

$$
\left.\frac{d}{d \epsilon}\right|_{\epsilon=0} \operatorname{Tr} T_{\epsilon}^{k}=0,\left.\quad \frac{1}{2} \frac{d^{2}}{d \epsilon^{2}}\right|_{\epsilon=0} \operatorname{Tr} T_{\epsilon}^{k}=(-1)^{k-1} k x y \omega^{k-1} .
$$

Proof. As in Lemma 2.1, we get

$$
\operatorname{Tr} T_{\epsilon}^{k}=(-1)^{k-1}\left(\epsilon^{2} x y+\omega\right)^{k} .
$$


The assertion follows from (5.10).

Lemma 5.6. Let $F(X)$ be a $G L(n+1, \mathbb{C})$-invariant polynomial. Then,

$$
\int_{\mathbb{P}^{1}} F\left(\frac{i}{2 \pi} R\right)=\frac{2 \pi i}{\omega}\left\{F\left(\frac{i}{2 \pi}\left(\begin{array}{cc}
\nu^{*} R_{E} & 0 \\
0 & 0
\end{array}\right)\right)-F(0)\right\} \text {. }
$$

Proof. Let $F\left(x_{1}, \cdots, x_{n+1}\right)$ be a polynomial such that $F(X)=F(\operatorname{Tr} X, \cdots$, $\left.\operatorname{Tr} X^{n+1}\right)$. By Lemma 5.5, we find

$$
\left.\frac{d}{d \epsilon}\right|_{\epsilon=0} F\left(t T_{\epsilon}\right)=0,\left.\quad \frac{d^{2}}{d \epsilon^{2}}\right|_{\epsilon=0} F\left(t T_{\epsilon}\right)=\frac{2 t x y}{\omega} \cdot \frac{d}{d t} F\left(\operatorname{Tr}\left(t T_{0}\right), \cdots, \operatorname{Tr}\left(t T_{0}\right)^{n+1}\right) .
$$

Since $x^{2}=y^{2}=0$, the coefficients of $\epsilon^{k}$ in the Taylor expansion of $F\left(t \cdot T_{\epsilon}\right)$ vanishes for $k>2$ and we have

$$
F\left(t T_{\epsilon}\right)=F\left(t T_{0}\right)+t \frac{x y}{\omega} \frac{d}{d t} F\left(t T_{0}\right) \epsilon^{2} .
$$

Set $t=\frac{i}{2 \pi} /\left(1+|v|^{2}\right)$ and $\epsilon=1$. Since

$$
t T_{1}=\frac{i}{2 \pi} R, \quad t T_{0}=\frac{i}{2 \pi} \frac{1}{1+|v|^{2}}\left(\begin{array}{cc}
\nu^{*} R_{E} & 0 \\
0 & 0
\end{array}\right), \quad t x y=\omega_{\mathbb{P}^{1}}
$$

where $\omega_{\mathbb{P}^{1}}$ is the Fubini-Study form of $\mathbb{P}^{1}$, we obtain

$$
\begin{aligned}
\int_{\mathbb{P}^{1}} F\left(\frac{i}{2 \pi} R\right) & =\left.\int_{\mathbb{P}^{1}} \omega_{\mathbb{P}^{1}} \frac{2 \pi i}{\omega} \frac{d}{d t}\right|_{t=\frac{1}{1+|v|^{2}}} F\left(t \frac{i}{2 \pi}\left(\begin{array}{cc}
\nu^{*} R_{E} & 0 \\
0 & 0
\end{array}\right)\right) \\
& =\frac{2 \pi i}{\omega} \int_{0}^{1} \frac{d}{d x} F\left(x \frac{i}{2 \pi}\left(\begin{array}{cc}
\nu^{*} R_{E} & 0 \\
0 & 0
\end{array}\right)\right) d x \\
& =\frac{2 \pi i}{\omega}\left\{F\left(\frac{i}{2 \pi}\left(\begin{array}{cc}
\nu^{*} R_{E} & 0 \\
0 & 0
\end{array}\right)\right)-F(0)\right\}
\end{aligned}
$$

Proof of Theorem 5.1. Putting $F(X)=T d(X)$ in Lemma 5.6, it follows from Lemma 2.2

$$
\int_{\mathbb{P}^{1}} T d\left(\frac{i}{2 \pi} R\right)=\frac{T d^{-1}\left(\nu^{*} c_{1}(H)\right)-1}{\nu^{*} c_{1}(H)} .
$$

In the same manner as $(5.14)$, we obtain

$$
\int_{\mathbb{P}^{1}} \log |v|^{2} T d\left(\frac{i}{2 \pi} R\right)=\frac{1}{\nu^{*} c_{1}(H)} \int_{0}^{1} d s \log \frac{1-s}{s} \frac{d}{d s} T d^{-1}\left(s \nu^{*} c_{1}(H)\right) .
$$

By (1.5) and (5.9), we have

$$
\widetilde{T d}(\overline{\mathcal{E}})=-\log \phi \int_{\mathbb{P}^{1}} T d\left(\frac{i}{2 \pi} R\right)-\int_{\mathbb{P}^{1}} \log |v|^{2} T d\left(\frac{i}{2 \pi} R\right),
$$

which combined with (5.15) and (5.16) yields the desired formula.

\section{Proof of main theorem for globalizable families.}

DEFINITION 6.1. Let $E$ be a holomorphic vector bundle on a complex manifold $X$. The pair $(X, E)$ is said to be globalizable, if $X$ is embeddable into a projective algebraic manifold of the same dimension to which $E$ extends as a coherent sheaf.

Let us use the same notations as in section 0 . 
Theorem 6.1. Suppose that the pair $(X, E)$ is globalizable. Then, $\|\cdot\|_{Q}$ is a singular Hermitian metric on $\lambda(E)$ whose curvature current is

$$
c_{1}\left(\lambda(E),\|\cdot\|_{Q}\right)=\frac{(-1)^{n+1}}{(n+2) !} r(E) \mu\left(\operatorname{Sing} X_{0}\right) \delta_{0}+\pi_{*}\left(T d\left(T X / S, g_{X / S}\right) \operatorname{ch}(E, h)\right)^{(1,1)}
$$

where $\pi_{*}(T d(T X / S) \operatorname{ch}(E, h))^{(1,1)}$ lies in $L_{\text {loc }}^{p}(S)$ for some $p>1$.

For the proof, we need the following Lemma.

Lemma 6.1. Let $D$ be the unit disc, $\chi \in L^{\infty}(D)$ and $f \in C^{\infty}(D)$. Suppose that $\Delta \chi=f$ on $D-\{0\}$ in the sense of distribution. Then $\chi \in C^{\infty}(D)$ and above equality holds on $D$ in the classical sense.

Proof. Since $f \in C^{\infty}(D)$, there exists $g \in C^{\infty}(D)$ such that $\Delta g=f$. Then $\Delta(\chi-g)=0$ on $D-\{0\}$ in the sense of distribution. As $\chi-g$ is bounded on a neighborhood of 0 , it extends to a harmonic function on $D$ by the Riemann extension theorem. Therefore $\chi$ is smooth on $D$ and satisfies $\Delta \chi=f$.

Proof of Theorem 6.1. The proof is similar to that of Bismut [B2]. At first, we treat the case that $X \subset X^{\prime}$ and $E$ extends to a vector bundle $E^{\prime}$ over $X^{\prime}$. By Theorem $1.2,1.3$ and 3.1 , we may asssume that the curvatures of $\left(T X, g_{X}\right)$ and $\left(E, h_{E}\right)$ vanish on a neighborhood of $\operatorname{Sing} X_{0}$ and that $g_{X}$ (resp. $h_{E}$ ) extends to a Kähler (resp. Hermitian) metric $g_{X^{\prime}}$ (resp. $h_{E^{\prime}}$ ) on $T X^{\prime}$ (resp. $E^{\prime}$ ). Since $\left\{X_{t}\right\}_{t \in S}$ is a family of divisors in $X^{\prime}, L:=\left\{L_{t}\right\}_{t \in S}\left(L_{t}:=\left[X_{t}\right]\right)$ is a holomorphic family of line bundles over $X^{\prime}$ and defines a line bundle over $X^{\prime} \times S$. Fix a smooth function $h$ on $S$ such that $h(u)=|u|^{2}$ for $|u|<\frac{1}{4}$ and $h(u)=1$ for $|u|>\frac{1}{2}$. Let $s \in H^{0}\left(X^{\prime} \times S, L\right)$ be the canonical section of $L$ such that $\left(s_{t}\right)_{0}=X_{t}$ where $s_{t}:=\left.s\right|_{X^{\prime} \times\{0\}}$. Define a Hermitian metric $h_{L_{t}}$ on $L_{t}$ such that $h_{L_{t}}\left(s_{t}, s_{t}\right)(z)=h(\pi(z)-t)$ for $z \in X$ and $h_{L_{t}}\left(s_{t}, s_{t}\right) \equiv 1$ for $z \in X^{\prime}-X$. Then, the family of metrics $h_{L}:=\left\{h_{L_{t}}\right\}$ becomes a smooth Hermitian metric on $L$. Put $\tilde{s}_{t}(z):=(\pi(z)-t)^{-1} s_{t}(z)$. Then $\tilde{s}_{t}$ is also a holomorphic section on a neighborhood of $X_{0}$ and trivializes $L_{t}$ over $X_{t}$. Moreover we get $h_{L_{t}}\left(\tilde{s}_{t}, \tilde{s}_{t}\right) \equiv 1$ on $X_{t}$. Let $d \pi$ be the generater of $N_{X_{t} / X}^{*}$ for any $t \in S-\{0\}$. Let $g_{N^{*}, t}^{\prime}$ be the Hermitian metric of $N_{X_{t} / X}^{*}$ defined by $g_{N^{*}, t}^{\prime}(d \pi, d \pi) \equiv 1$. Put $g_{N, t}^{\prime}:=\left(g_{N^{*}, t}^{\prime}\right)^{-1}$. Then on $X_{0}$ holds the following equality by the construction of $h_{L_{t}}$ and $g_{N^{*}, t}^{\prime}$ :

$$
\left\|d \pi \otimes \tilde{s}_{t}\right\|_{g_{N^{*}, t}^{\prime} \otimes L_{t}} \equiv 1
$$

Let $\sigma_{0} \in \Gamma\left(S, \lambda_{X}(E)\right)$ be a holomorphic section such that $\sigma(t) \neq 0$ for any $t \in S$. Let $p_{i}:=p r_{i}$ be the projection to the $i$-th factor in $X^{\prime} \times S$. Regarding $X^{\prime} \times S$ as the trivial family over $S$, we obtain line bundles $\lambda_{X^{\prime} \times S}\left(p_{1}^{*} E^{\prime}\right)$ and $\lambda_{X^{\prime} \times S}\left(p_{1}^{*} E^{\prime} \otimes L^{-1}\right)$ over $S$. By the construction of $L, \lambda:=\lambda_{X}(E) \otimes \lambda_{X^{\prime} \times S}\left(p_{1}^{*} E^{\prime}\right)^{-1} \otimes \lambda_{X^{\prime} \times S}\left(p_{1}^{*} E^{\prime} \otimes L\right)$ is canonically trivial and has a canonical section $\sigma$. Let $\sigma_{1} \in \Gamma\left(S, \lambda_{X^{\prime} \times S}\left(p_{1}^{*} E^{\prime}\right)\right)$ and $\sigma_{2} \in \Gamma\left(S, \lambda_{X^{\prime} \times S}\left(p_{1}^{*} E^{\prime} \otimes L^{-1}\right)\right)$ be nowhere vanishing sections such that

$$
\sigma_{0} \otimes \sigma_{1}^{-1} \otimes \sigma_{2}=\sigma
$$

Let $\|\cdot\|_{Q 1}$ and $\|\cdot\|_{Q 2}$ be the Quillen metrics of $\lambda_{X^{\prime} \times S}\left(p_{1}^{*} E^{\prime}\right)$ and $\lambda_{X^{\prime} \times S}\left(p_{1}^{*} E^{\prime} \otimes\right.$ $\left.L^{-1}\right)$ relative to $\left(g_{X^{\prime}}, h_{E^{\prime}}\right)$ and $\left(g_{X^{\prime}}, h_{E^{\prime}} \otimes h_{L}^{-1}\right)$ respectively. By $(6.1)$, we may apply Theorem 1.4 to $X_{t} \subset X^{\prime}$ to obtain

$$
\log \left\|\sigma_{0}\right\|^{2}(t)_{Q}=\left[\int_{X_{t}} \widetilde{T d}\left(\overline{\mathcal{E}}_{t}\right) \operatorname{ch}\left(E^{\prime}, h_{E}\right)\right]^{(0,0)}+\phi(t)
$$


where

$$
\begin{aligned}
\phi(t)= & \log \left\|\sigma_{1}\right\|_{Q 1}^{2}(t)-\log \left\|\sigma_{2}\right\|_{Q 2}^{2}(t) \\
& -\int_{X^{\prime}} T d\left(T X^{\prime}, g_{X^{\prime}}\right) T d^{-1}\left(L_{t}, h_{L_{t}}\right) \operatorname{ch}\left(E^{\prime}, h_{E^{\prime}}\right) \log \left\|s_{t}\right\|_{L_{t}}^{2}+\text { Const } .
\end{aligned}
$$

and $\overrightarrow{\mathcal{E}}$ is an exact sequence of holomorphic Hermitian vector bundles:

$$
\overline{\mathcal{E}}_{t}: 0 \longrightarrow\left(T X_{t}, g_{X_{t}}\right) \longrightarrow\left(\left.T X\right|_{X_{t}}, g_{X}\right) \longrightarrow\left(N_{X_{t} / X^{\prime}}, g_{N, t}^{\prime}\right) \longrightarrow 0 .
$$

Since $\log \left\|\sigma_{1}\right\|_{Q 1}^{2}$ and $\log \left\|\sigma_{2}\right\|_{Q 2}^{2}$ are smooth function on $S$ by Theorem $1.1, \phi$ is a continuous function on $S$. Let $U_{p}$ be a small neighborhood of $p \in \operatorname{Sing} X_{0}$ in $X$. As $\widetilde{T d}\left(\overline{\mathcal{E}}_{t}\right)$ depends smoothly on $t \in S$ over $X-\cup_{p \in \operatorname{Sing}} X_{0} U_{p}$ and $\left(E^{\prime}, h_{E^{\prime}}\right)$ is flat on each $U_{p}$, we get

$$
\log \left\|\sigma_{0}\right\|_{Q}^{2}(t)=r(E) \sum_{p \in \text { Sing } X_{0}}\left[\int_{X_{t} \cap U_{p}} \widetilde{T d}\left(\overline{\mathcal{E}}_{t}\right)\right]^{(0,0)}+\psi(t)
$$

for some $\psi \in C^{0}(S)$. Put $f_{p}:=\left.\pi\right|_{U_{p}}$. By Theorem 4.1 and 5.1,

$$
\begin{aligned}
\int_{X_{t} \cap U_{p}} \widetilde{T d}\left(\overrightarrow{\mathcal{E}}_{t}\right) & =\int_{X_{t} \cap U_{p}} \frac{1-T d^{-1}\left(\nu_{f_{p}}^{*} c_{1}(H)\right)}{\nu_{f_{p}}^{*} c_{1}(H)} \log \left\|d f_{p}\right\|^{2}+c(n) \int_{X_{t} \cap U_{p}} \nu_{f_{p}}^{*} c_{1}(H)^{n} \\
& =\frac{(-1)^{n+1}}{(n+2) !} \int_{X_{t} \cap U_{p}}\left(\frac{i}{2 \pi} \partial \vec{\partial} \log \left\|d f_{p}\right\|^{2}\right)^{n} \log \left\|d f_{p}\right\|^{2}+O(1) \\
& =\frac{(-1)^{n+1}}{(n+2) !} \mu(p) \log |t|^{2}+O(1)
\end{aligned}
$$

which together with (6.6) yields

$$
\log \left\|\sigma_{0}\right\|_{Q}^{2}(t)=\frac{(-1)^{n+1}}{(n+2) !} r(E) \mu(p) \log |t|^{2}+\chi(t), \quad \chi \in L^{\infty}(S) .
$$

By Theorem 1.1, it holds on $S-\{0\}$ that

$$
\frac{i}{2 \pi} \bar{\partial} \partial \chi=\pi_{*}\left(T d\left(T X / S, g_{X / S}\right) \operatorname{ch}(E, h)\right)^{(1,1)} .
$$

Since $\pi_{*}\left(T d\left(T X / S, g_{X / S}\right) \operatorname{ch}(E, h)\right)^{(1,1)}$ extends to a smooth $(1,1)$-form over $S$ by Proposition 2.1 and $\chi \in L^{\infty}(S)$, we find by (6.9) and Lemma 6.1 that $\chi \in C^{\infty}(S)$. The assertion follows from (6.8), (6.9) and smoothness of $\chi$.

Next, we treat the general case where the extension $E^{\prime}$ of $E$ is no more a vector bundle. Since $X^{\prime}$ is a projective manifold, there exists a resolution of $E^{\prime}$ by vector bundles $\left\{E_{i}\right\}_{1 \leq i \leq N}$ over $X^{\prime}$ :

$$
0 \rightarrow E_{N} \rightarrow E_{N-1} \rightarrow \cdots \rightarrow E_{1} \rightarrow E^{\prime} \rightarrow 0 .
$$

Here we identify vector bundles with corresponding locally free sheaves. Fix Hermitian metrics $\left\{h_{i}\right\}$ on each $E_{i}$. As every $E_{i}$ is a vector bundle, we can apply Theorem 6.1 for each $\left(E_{i}, h_{i}\right)$ over $X$. Since $E^{\prime}$ is a vector bundle over $X$, by an argument using anomaly formula ([B-G-S, Theorem 0.3]), we also get the theorem for $(E, h)$.

7. Approximation by algebraic families and proof of main theorem. We prove Main Theorem in this section. For simplicity, we assume that $\operatorname{Sing} X_{0}$ consists of one point i.e., Sing $X_{0}=\{0\}$. (General case is proved similarly.) 
Step 1. Fix an embedding $i: X \hookrightarrow \mathbb{P}^{N} \times S, \pi=p r_{2} \circ i$ and define the polarization $\mathcal{O}_{X}(1):=i^{*} \operatorname{pr}_{1}^{*} \mathcal{O}_{\mathbb{P}^{N}}(1)$. Since $X$ is projective over $S$, it is enough to show the theorem under the assumption that the vector bundle $E$ is a quotient of some $\mathcal{O}_{X}(-1)^{\oplus l}$ : $\psi: \mathcal{O}_{X}(-1)^{\oplus l} \rightarrow E \rightarrow 0$. Let $P_{s}(m)$ be the Hilbert polynomial of $X_{s}(s \in S)$ with respect to $\mathcal{O}_{X}(1)$. As $X$ is flat over $S, P(m)=P_{s}(m)$ is constant in $s \in S$. Let $T:=H i l b_{\mathbb{P} N}^{P}$ be the Hilbert scheme of subvarieties with the Hilbert polynomial $P(m)$ and $p_{1}: Y \rightarrow T$ the universal family over $T$. Then, $Y \subset T \times \mathbb{P}^{N}$ and $Y_{t}$ is a subvariety of $\mathbb{P}^{N}$ corresponding to $t \in \operatorname{Hilb}_{\mathbb{P}^{N}}^{P}$ (cf $\left.[\mathrm{G}]\right)$. Set $\mathcal{O}_{Y}(1):=\left.\mathcal{O}_{\mathbb{P}^{N}}(1)\right|_{Y}$. Let $\phi_{s}(m)$ be the Hilbert polynomial of $\mathcal{O}_{X_{s}}\left(E_{s}\right)$ with respect to $\mathcal{O}_{X_{s}}(1)$. Since $\mathcal{O}_{X}(E)$ is flat over $S, \phi(m)=\phi_{s}(m)$ is constant in $s \in S$. Let $Q=\operatorname{Quot}^{\phi}\left(\mathcal{O}_{Y}(-1)^{\oplus l} / Y / T\right)$ be the Quot-scheme of coherent $\mathcal{O}_{Y}$-modules which are quotient of $\mathcal{O}_{Y}(-1)^{\oplus l}$ with the Hilbert polynomial $\phi(m)$. Let $p_{2}: Q \rightarrow T$ be the projection. Put $Y_{Q}:=Y \times_{T} Q$ and $q: Y_{Q} \rightarrow Q$ for the projection to the second factor. The universal quotient sheaf over $Y_{Q}$ is denoted by $F$ (cf. [G]): $\Psi: \mathcal{O}_{Y_{Q}}(-1)^{\oplus l} \rightarrow F \rightarrow 0$.

Let $\gamma: S \rightarrow Q$ be the holomorphic map defined by $\gamma(s)=\left(X_{s}, E_{s}\right) \in Q$. Then, by the universality of the Hilbert scheme and the Quot-scheme, we get the following identification:

$$
X=Y_{Q} \times_{\gamma} S, \quad E=\gamma^{*} F .
$$

Since $T$ and $Q$ are projective varieties, we can approximate the holomorphic curve $\gamma: S \rightarrow Q$ by a sequence of algebraic curves due to Demailly, Lempert and Schiffman ([D-L-S], [L]).

LEMMA 7.1. There exist a sequence of algebraic curves $\left\{C_{m}\right\}_{m \geq 1}$ with distinguished open subsets $\left\{U_{m}\right\}_{m \geq 1}$ isomorphic to $S$, i.e., $i_{m}: S \cong U_{m}$, and a sequence of holomorphic maps $\gamma_{m}: C_{m} \rightarrow Q$ such that, under the identification of $U_{m}$ with $S$ via $i_{m}$

(1) $\gamma_{m}(t)=\gamma(t)+O\left(t^{m+1}\right)$,

(2) $\gamma_{m}$ converges to $\gamma$ uniformly on $S\left(\frac{1}{2}\right)$.

Put $X_{m}:=Y_{Q} \times_{\gamma_{m}} S, \pi_{m}:=\left.p r_{2}\right|_{X_{m}}$ and $E_{m}:=\gamma_{m}^{*} F$. Then, the pair $\left(X_{m}, E_{m}\right)$ is globalizable for any $m$. To show this, put $Z_{m}:=Y_{Q} \times_{\gamma_{m}} C_{m}, E_{m}^{\prime}:=\gamma_{m}^{*} F$. Let $f_{m}: \tilde{Z}_{m} \rightarrow Z_{m}$ be a resolution of singularity and put $E_{m}^{\prime \prime}:=f_{m}^{*} E_{m}^{\prime}$. Since $X$ is non-singular, so is $X_{m}$ by the construction. Thus, we may assume that $f_{m}$ is an isomorphism restricted to $f_{m}^{-1}\left(X_{m}\right)$. As $Y_{Q}$ is projective, so is $\tilde{Z}_{m}$. Furthermore, as $F$ is a coherent sheaf over $Y_{Q}$, so is $E_{m}^{\prime \prime}$ over $\tilde{Z}_{m}$. Clearly, $\left(\tilde{Z}_{m}, E_{m}^{\prime \prime}\right)$ is a globalization of $\left(X_{m}, E_{m}\right)$.

Step 2. Let $\left(Y_{Q}, 0\right)$ and $(Q, \gamma(0))$ be the germs of analytic spaces at 0 and $\gamma(0)$ respectively. Since $q: Y_{Q} \rightarrow Q$ is a flat morphism, $q:\left(Y_{Q}, 0\right) \rightarrow(Q, \gamma(0))$ becomes a deformation of isolated hypersurface singularity $\left(X_{0}, 0\right)$. Since $\left(X_{0}, 0\right)$ is an IHS, its Kuranishi space $\operatorname{De} f\left(X_{0}, 0\right)$ is non-singular and $\operatorname{Def}\left(X_{0}, 0\right)=\left(\mathbb{C}^{\mu}, 0\right)$ where $\mu$ is the Milnor number of $\left(X_{0}, 0\right)$. Let $w=\left(w_{0}, \cdots, w_{n}\right)$ be the coordinates of $\left(\mathbb{C}^{n+1}, 0\right)$ and $t=\left(t_{1}, \cdots, t_{\mu}\right)$ of $\operatorname{Def}\left(X_{0}, 0\right)$. The semi-universal deformation of $\left(X_{0}, 0\right)$ is constructed as follows (cf. [Lo, Chap.6]). Let $f(w)$ be a polynomial in $w$-variables such that $\mathcal{O}_{X_{0}, 0} \cong \mathbb{C}\{w\} /(f(w))$ and $\left\{\phi_{1}(w), \cdots, \phi_{\mu}(w)\right\}$ a basis of the $\mathbb{C}$-vector space $\mathbb{C}\{w\} /\left(\partial f / \partial w_{0}, \cdots, \partial f / \partial w_{n}\right)$. Then,

$$
(\mathcal{X}, 0):=\left\{(w, t) \in \mathbb{C}^{n+1} \times \operatorname{Def}\left(X_{0}, 0\right) ; f(w)+\sum_{i=1}^{\mu} t_{i} \phi_{i}(w)=0\right\}
$$

with the projection map $p r_{2}:(\mathcal{X}, 0) \rightarrow \operatorname{Def}\left(X_{0}, 0\right)$ becomes the semi-universal de- 
formation. By the versality, there exists a small neighborhood of 0 in $Y_{Q}$, a small neighborhood $V$ of $\gamma(0)$ in $Q$, a holomorphic map $J:(V, \gamma(0)) \rightarrow \operatorname{Def}\left(X_{0}, 0\right)$ and an embedding $j:(U, 0) \hookrightarrow(\mathcal{X}, 0) \times_{J}(V, \gamma(0))$ such that

(1) $q((U, 0))=(V, \gamma(0)),\left.q\right|_{U}=p r_{2} \circ j$

(2) $j:\left(X_{0} \cap U, 0\right) \hookrightarrow\left(\mathcal{X}_{0}, 0\right)$ is an embedding.

Since $(\mathcal{X}, 0) \times_{J}(V, \gamma(0)) \subset\left(\mathbb{C}^{n+1}, 0\right) \times(V, \gamma(0))$, we may consider $j$ to be an embedding: $j:(U, 0) \hookrightarrow\left(\mathbb{C}^{n+1}, 0\right) \times(V, \gamma(0))$.

Step 3. Let us construct a good metric on the relative tangent bundle $T Y_{Q} / Q$. Since $Y_{Q} \subset \mathbb{P}^{N} \times Q$, there exists another embedding $i:(U, 0) \hookrightarrow\left(\mathbb{P}^{N}, 0\right) \times(V, \gamma(0))$. Fix an embedding $k:(V, \gamma(0)) \hookrightarrow\left(\mathbb{C}^{M}, 0\right)$. Let $u=\left(u_{1}, \cdots, u_{M}\right)$ be the coordinates of $\mathbb{C}^{M}$. Then, the embedding induces a Kähler metric on $V$ by $d s_{V}^{2}:=k^{*} d s_{\mathbb{C}^{M}}^{2}$ where $d s_{\mathbb{C}^{M}}^{2}=\partial \bar{\partial}\|u\|^{2}$ is the Euclidean metric of $\mathbb{C}^{M}$. By the embeddings $i$ and $j, U$ is eqquiped with the following Kähler metrics:

$$
g_{1}:=i^{*}\left(d s_{\mathbb{P}^{N}}^{2}+d s_{V}^{2}\right), \quad g_{2}:=j^{*}\left(d s_{\mathbb{C}^{n+1}}^{2}+d s_{V}^{2}\right)
$$

where $d s_{\mathbb{P}^{N}}^{2}$ is the Fubini-Study metric. Since $g_{1}$ and $g_{2}$ are induced by embeddings into some ambient spaces, they are quasi-isometric to each other. Namely, there exists a constant $C>0$ such that it holds on $U$

$$
C^{-1} g_{1} \leq g_{2} \leq C g_{1} \text {. }
$$

Let $z=\left(z_{1}, \cdots, z_{N}\right)$ be the inhomogeneous coordinates of $\mathbb{P}^{N}$ centered at 0 . Let $B(r)$ be the metric balll of radius $r$ centered at 0 in $\mathbb{P}^{N}$ and $U(r):=U \cap(B(r) \times V)$. Fix $\delta>0$ such that $U(\delta) \subset U$. Let $\rho_{1}(r) \in C^{\infty}(\mathbb{R})$ such that $\rho_{1}(r) \equiv 0$ for $r \leq \delta_{1}$, $\rho_{1}(r)=r$ for $r \geq \delta_{2}, \rho_{1}^{\prime}(r) \geq 0$ and $\rho_{1}^{\prime \prime}(r) \geq 0$ for any $r \in \mathbb{R}$ where $0<\delta_{1}<\delta_{2}<\delta$. Let $\rho_{2}(r)$ be a smooth cut-off function such that $\rho_{2}(r) \equiv 1$ for $r \leq \delta_{2}$ and $\rho_{2}(r) \equiv 0$ for $r \geq \delta$. Define a potential function $\Phi_{\epsilon} \in C^{\infty}(U)$ by

$$
\Phi_{\epsilon}:=\epsilon i^{*} \rho_{2}\left(\log \left(1+\|z\|^{2}\right)\right) j^{*}\left(\|w\|^{2}+\|u\|^{2}\right)+i^{*}\left\{\rho_{1}\left(\log \left(1+\|z\|^{2}\right)\right)+\|u\|^{2}\right\} .
$$

Since $d s_{\mathbb{P}^{N}}^{2}=\partial \bar{\partial} \log \left(1+\|z\|^{2}\right)$ and $d s_{\mathbb{C}^{n+1}}^{2}=\partial \bar{\partial}\|w\|^{2}$, we find by (7.4) that $\partial \bar{\partial} \Phi_{\epsilon}>0$ on $U$ if $\epsilon$ is sufficiently small. By the construction,

$$
\left.\partial \bar{\partial} \Phi_{\epsilon}\right|_{T Y_{Q} / Q}(x)= \begin{cases}\left.\epsilon j^{*} d s_{\mathbb{C}^{n+1}}^{2}\right|_{T Y_{Q} / Q} & \left(x \in U\left(\delta_{1}\right)\right) \\ \left.i^{*} d s_{\mathbb{P}^{N}}^{2}\right|_{T Y_{Q} / Q} & \left(x \in U \backslash U\left(\delta_{0}\right)\right) .\end{cases}
$$

Therefore, we can define a Hermitian metric $g_{Y_{Q} / Q}$ on $T Y_{Q} / Q$ by

$$
g_{Y_{Q} / Q}(x)= \begin{cases}\left.\partial \bar{\partial} \Phi_{\epsilon}\right|_{T Y_{Q} / Q}(x) & (x \in U) \\ \left.i^{*} d s_{\mathbb{P}^{N}}^{2}\right|_{T Y_{Q} / Q}(x) & \left(x \in U \backslash U\left(\delta_{0}\right)\right) .\end{cases}
$$

By Proposition 2.1 and (7.6), we get the following.

LEMMA 7.2. It holds on $U\left(\delta_{1}\right)$ that $\left[T d\left(T Y_{Q} / Q, g_{Y_{Q} / Q}\right)\right]^{(n+1, n+1)} \equiv 0$. Moreover, $\left[T d\left(T Y_{Q} / Q, g_{Y_{Q} / Q}\right)\right]^{(n+1, n+1)}$ extends to a smooth $(n+1, n+1)$-form on a neighborhood of $X_{0}$.

Step 4. Let us construct a good metric on $F$. Since $F$ is locally free along $X_{0}$, so is on a neighborhood of $X_{0}$ in $Y_{Q}$ because locally freeness is an open condition. In particular, there exists a hermitian metric $h_{F}$ on $\left.F\right|_{q^{-1}(V)}$ whose curvature vanishes on $U$ if $U$ and $V$ are chosen small enough.

Step 5. Let $g_{m}:=\gamma_{m}^{*} g_{Y_{Q} / Q}$ and $h_{m}:=\gamma_{m}^{*} h_{F}$ be the induced Hermitian metrics on $T X_{m} / S$ and $E_{m}$. Since it is enough to show Main Theorem for some Kähler metric of $X$ and some Hermitian metric of $E$, we may assume that $T X / S$ and $E$ are 
equipped with the restriction of $g_{Y_{Q} / Q}$ and $h_{F}$. Let $\lambda=\operatorname{det} R p_{*} F$ be the determinant of cohomologies, $\|\cdot\|_{Q, m}$ the Quillen metric of $\left.\lambda\right|_{X_{m}}$ relative to $g_{m}$ and $h_{m}$. Let $\sigma \in \Gamma(U, \lambda)$ be a holomorphic section such that $\sigma(z) \neq 0$ for any $z \in U$. Applying Theorem 6.1 for $\left\{\left(X_{m}, g_{m}\right),\left(E_{m}, h_{m}\right)\right\}$, we get the following formula.

$c_{1}\left(\left.\gamma_{m}^{*} \lambda\right|_{X_{m}},\|\cdot\|_{Q, m}\right)=\frac{(-1)^{n+1}}{(n+2) !} \mu\left(X_{0}\right) r(E) \delta_{0}+\pi_{*}\left(T d\left(T X_{m} / S, g_{m}\right) \operatorname{ch}\left(E_{m}, h_{m}\right)\right)^{(1,1)}$.

By (7.10) and Lemma 7.2, there exist a family of smooth functions $\left\{\chi_{m}\right\} \subset C^{\infty}(S)$ such that, on $S$,

$$
\log \left\|\gamma_{m}^{*} \sigma\right\|_{Q, m}^{2}(t)=\frac{(-1)^{n}}{(n+2) !} \mu\left(X_{0}\right) r(E) \log |t|^{2}+\chi_{m}(t)
$$

From Lemma 7.1 and the construction of metrics $g_{Y_{Q} / Q}$ and $h_{F}$, it follows that for any $t \in S-\{0\}$,

$$
\left\|\gamma_{m}^{*} \sigma\right\|_{Q, m}(t) \rightarrow\left\|\gamma^{*} \sigma\right\|_{Q}(t) \quad(m \rightarrow \infty),
$$

because $\log \|\sigma\|_{Q}^{2}$ is a smooth function on $V \backslash \Delta$ where $\Delta$ is the discriminant locus of $q: Y_{Q} \rightarrow Q$. In particular, the family $\left\{\chi_{m}\right\}$ is uniformly bounded on $\partial S\left(\frac{1}{2}\right)$. By (7.9) and Theorem 1.1, we have

$$
\frac{i}{2 \pi} \bar{\partial} \partial \log \chi_{m}=\pi_{*}\left(T d\left(T X_{m} / S, g_{m}\right) \operatorname{ch}\left(E_{m}, h_{m}\right)\right)^{(1,1)} .
$$

Since $\left\{\pi_{*}\left(T d\left(T X_{m} / S, g_{m}\right) \operatorname{ch}\left(E_{m}, h_{m}\right)\right)^{(1,1)}\right\}$ is a family of smooth $(1,1)$-forms on $S\left(\frac{1}{2}\right)$ converging uniformly to $\pi_{*}\left(T d\left(T X / S, g_{X / S}\right) \operatorname{ch}(E, h)\right)^{(1,1)}$ by Lemma 7.2 and Step 4, it follows from the elliptic regularity theory (cf. [G-T, Theorem 8.6]) that $\left\{\chi_{m}\right\}$ is an uniformly bounded equi-continuous family on $S\left(\frac{1}{2}\right)$. Put

$$
\chi(t):=\log \left\|\gamma^{*} \sigma\right\|_{Q}^{2}(t)-\frac{(-1)^{n}}{(n+2) !} \mu\left(X_{0}\right) r(E) \log |t|^{2} .
$$

From (7.10) together with the Ascoli-Arzelá Theorem, it follows that $\lim \chi_{m}=\chi$ uniformly on $S\left(\frac{1}{2}\right)$ and $\chi \in C^{\infty}\left(S\left(\frac{1}{2}\right)\right)$. Main Theorem follows from (7.12) and the smoothness of $\chi$.

8. Smoothing of IHS and analytic torsion. We prove Theorem 0.1 in this section. The same notations as in section 0 are used through this section. First, we study the behavior of the $L^{2}$-metric on $\lambda\left(\mathcal{O}_{X}\right)$.

Let $H$ be the relatively very ample line bundle over $X$. We may assume that $X \subset \mathbb{P}^{N} \times S, H=p r_{1}^{*} \mathcal{O}_{\mathbb{P}^{N}}(1)$ and $\pi=\left.p r_{2}\right|_{X}$ where $p r_{i}$ is the projection to the $i$-th factor. Since $\operatorname{Sing} X_{0}$ is a finite set, we can choose generic hyperplane sections $H_{1}, \cdots, H_{n}$ such that $\operatorname{Sing} X_{0} \notin H_{i}$ and $\operatorname{Sing}\left(X_{0} \cap H_{1} \cap \cdots \cap H_{i}\right)=\emptyset$ for any $i=1, \cdots, n$. Put $X^{q}:=X \cap H_{1} \cap \cdots \cap H_{n-q}, \pi^{q}:=\left.\pi\right|_{X^{q}}$ and $X_{t}^{q}:=X^{q} \cap X_{t}=$ $X_{t} \cap H_{1} \cap \cdots \cap H_{q}$. Then, we may also assume that $\pi^{q}: X^{q} \rightarrow S$ is a smooth morphism for any $q>0$ by the construction. As was shown by Steenbrink ([St]), $R^{q} \pi_{*} \mathcal{O}_{X}$ is a locally free $\mathcal{O}_{S}$-module for any $q \geq 0$. Choose a $\mathcal{O}_{S}$-basis $\omega_{1}^{q}, \cdots, \omega_{m_{q}}^{q}$ of $R^{q} \pi_{*} \mathcal{O}_{X}$. Put $\sigma^{q}:=\wedge_{i=1}^{m_{q}} \omega_{i}^{q} \in \operatorname{det} R^{q} \mathcal{O}_{X_{t}}$ and $\sigma:=\otimes_{q=0}^{n}\left(\sigma^{q}\right)^{(-1)^{q}} \in \lambda\left(\mathcal{O}_{X}\right)$. By Dolbeaut's theorem, there exists $\bar{\partial}$-closed $(0, q)$-form $\phi_{i}^{q}$ representing $\omega_{i}^{q}$ defined on $X_{\epsilon}:=\pi^{-1}\left(S_{\epsilon}\right)$ where $S_{\epsilon}=\{t \in S ;|t| \leq \epsilon\}$. Put $\phi_{i}^{q}(t):=\left.\phi_{i}^{q}\right|_{X_{t}}$ and $\psi_{i}^{q}(t):=\left.\phi_{i}^{q}\right|_{X_{t}^{q}}$. Let $\mathbb{H} \phi_{i}^{q}(t)$ and $\mathbb{H} \psi_{i}^{q}(t)$ 
be their harmonic representatives. Then, $\overline{\mathbb{H} \phi_{i}^{q}(t)}$ (resp. $\overline{\mathbb{H} \psi_{i}^{q}(t)}$ ) is a holomorphic $q$-from on $X_{t}$ (resp. $\left.X_{t}^{q}\right)$. In particular, $\left.\mathbb{H} \phi_{i}^{q}(t)\right|_{X_{t}^{q}}$ is also a harmonic form on $X_{t}^{q}$.

LEMma 8.1.

$$
\left.\mathbb{H} \phi_{i}^{q}(t)\right|_{X_{t}^{q}}=\mathbb{H} \psi_{i}^{q}(t) .
$$

Proof. Let $\left[\mathbb{H} \phi_{i}^{q}(t)\right]\left(\right.$ resp. $\left.\left[\mathbb{H} \psi_{i}^{q}(t)\right]\right)$ be the Dolbeaut cohomology class. Then, we get the assertion by

$$
\left[\left.\mathbb{H} \phi_{i}^{q}(t)\right|_{X_{t}^{q}}\right]=\left.\left[\mathbb{H} \phi_{i}^{q}(t)\right]\right|_{X_{t}^{q}}=\left.\left[\phi_{i}^{q}\right]\right|_{X_{t}^{q}}=\left[\mathbb{H} \psi_{i}^{q}(t)\right]
$$

because both $\left.\mathbb{H} \phi_{i}^{q}(t)\right|_{X_{t}^{q}}$ and $\mathbb{H} \psi_{i}^{q}(t)$ are harmonic forms on $X_{t}^{q}$.

Proposition 8.1. $\left\|\sigma^{q}\right\|_{L^{2}}^{2}$ is a positive $C^{\infty}$-function on $S$ for any $q<n$.

Proof. Let $g_{t}$ be the Kähler form of $\left.g_{X}\right|_{X_{t}}$ and $\left[g_{t}\right]$ its Kähler class. By the assumption, we get $\left[g_{t}\right]=\left.c_{1}(H)\right|_{X_{t}}$ in the de Rham cohomology group. From the definition of $L^{2}$-metric and Lemma 8.1, it follows that

$$
\begin{aligned}
\left\|\sigma^{q}\right\|_{L^{2}}^{2}(t) & =\left|\operatorname{det}\left(\int_{X_{t}} \mathbb{H} \phi_{i}^{q}(t) \wedge \overline{\mathbb{H} \phi_{j}^{q}}(t) \wedge g_{t}^{n-q}\right)_{1 \leq i, j \leq m_{q}}\right| \\
& =\left|\operatorname{det}\left(\int_{X_{t}} \mathbb{H} \phi_{i}^{q}(t) \wedge \overline{\mathbb{H} \phi_{j}^{q}}(t) \wedge c_{1}(H)^{n-q}\right)_{1 \leq i, j \leq m_{q}}\right| \\
& =\left|\operatorname{det}\left(\int_{X_{t}^{q}} \mathbb{H} \psi_{i}^{q}(t) \wedge \overline{\mathbb{H} \psi_{j}^{q}}(t)\right)_{1 \leq i, j \leq m_{q}}\right| .
\end{aligned}
$$

Therefore, it is enough to show that $\left.\left[\phi_{1}^{q}\right]\right|_{X_{0}^{q}}, \cdots,\left.\left[\phi_{m_{q}}^{q}\right]\right|_{X_{0}^{q}}$ are linearly independent in $H^{q}\left(X_{0}^{q}, \mathcal{O}_{X_{0}^{q}}\right)$ to prove the proposition. Since $H_{1}$ is an ample divisor of $X_{0}$, $r_{1}: H^{q}\left(X_{0}, \mathcal{O}_{X_{0}}\right) \rightarrow H^{q}\left(X_{0}^{1}, \mathcal{O}_{X_{0}^{1}}\right)$ is an isomorphism for $q<n-1$ and an injection for $q=n-1$ where $r_{1}$ is the restriction map. Inductively, we find that the restriction map $r_{q}: H^{q}\left(X_{0}, \mathcal{O}_{X_{0}}\right) \rightarrow H^{q}\left(X_{0}^{q}, \mathcal{O}_{X_{0}^{q}}\right)$ is an injection for any $q<n$. In particular, $\left.\left[\phi_{1}^{q}\right]\right|_{X_{0}^{q}}, \cdots,\left.\left[\phi_{m_{q}}^{q}\right]\right|_{X_{0}^{q}}$ are linearly independent in $H^{q}\left(X_{0}^{q}, \mathcal{O}_{X_{0}^{q}}\right)$ because so are $\left.\left[\phi_{1}^{q}\right]\right|_{X_{0}}, \cdots,\left.\left[\phi_{m_{q}}^{q}\right]\right|_{X_{0}}$ in $H^{q}\left(X_{0}, \mathcal{O}_{X_{0}}\right)$ by the linearly independence of $\omega_{1}^{q}, \cdots, \omega_{m_{q}}^{q}$ at $t=0$.

Let us study the case $q=n$. Write $\phi_{i}^{n}=\tau_{i}$ and $m_{n}=m$. Let $\omega_{1}, \cdots, \omega_{m}$ be the basis of $\omega_{X / S}$ as in section 0 . We can choose $\tau_{i}$ such that

$$
\int_{X_{t}} \omega_{i} \wedge \tau_{j}=\delta_{i j}
$$

Proposition 8.2.

$$
\left|\operatorname{det}\left(\int_{X_{t}} \mathbb{H} \tau_{i}(t) \wedge \mathbb{H} \bar{\tau}_{j}(t)\right)_{1 \leq i, j \leq m}\right|=\operatorname{det}\left({ }^{t} \Omega(t) \Lambda \bar{\Omega}(t)\right)^{-1} .
$$

Proof. Write $\mathbb{H} \tau_{i}(t)=\sum_{j=1}^{m} a_{i j}(t) \bar{\omega}_{j}(t)$ for some $(m, m)$-matrix $A(t):=\left(a_{i j}(t)\right)$. Then we have

$$
\left|\operatorname{det}\left(\int_{X_{t}} \mathbb{H} \tau_{i}(t) \wedge \mathbb{H}_{j}(t)\right)_{1 \leq i, j \leq m}\right|=|\operatorname{det} A(t)|^{2} \cdot\left|\operatorname{det}\left(\int_{X_{t}} \omega_{i}(t) \wedge \bar{\omega}_{j}(t)\right)_{1 \leq i, j \leq m}\right| .
$$


By (8.3), we find

$$
A(t)=\left(\int_{X_{t}} \omega_{i}(t) \wedge \bar{\omega}_{j}(t)\right)^{-1}
$$

Let $\left\{a_{1}(t), \cdots, a_{l}(t)\right\}$ be the basis of $H_{n}\left(X_{t}, \mathbb{Z}\right)_{f r}$ as in section 0 . By the Küneth formula for $\Delta_{t} \subset X_{t} \times X_{t}$ where $\Delta_{t}$ is the diagonal set, it holds in $H_{n}\left(X_{t}, \mathbb{Z}\right)_{f r}$

$$
\left[\Delta_{t}\right]=\sum_{i, j=1}^{m} \lambda_{i j} p r_{1}^{*} a_{i}(t) \wedge p r_{2}^{*} a_{j}(t)+E
$$

where $E$ is the term containing an element of $p r_{i}^{*} H_{q}\left(X_{t}, \mathbb{Z}\right)_{f r}(q \neq n)$ and $\Lambda=\left(\lambda_{i j}\right)$ is the intersection form on $H_{n}\left(X_{t}, \mathbb{Z}\right)_{f r}$. By (8.6), we get

$$
\begin{aligned}
\int_{X_{t}} \omega_{i}(t) \wedge \bar{\omega}_{j}(t) & =\int_{\Delta_{t}} p r_{1}^{*} \omega_{i}(t) \wedge p r_{2}^{*} \bar{\omega}_{j}(t) \\
& =\sum_{i, j=1}^{m} \lambda_{i j} \int_{a_{i}(t)} \omega_{i}(t) \overline{\int_{a_{j}(t)} \omega_{j}(t)}
\end{aligned}
$$

which together with (8.4) and (8.5) yields the assertion.

PropositTon $8.3([\mathrm{M}])$. The following asymptotic expansion holds as $t \rightarrow 0$ :

$$
\int_{a_{i}(t)} \omega_{j}(t) \sim \sum_{r=r_{1}, \cdots, r_{k}} \sum_{i \geq 0} \sum_{j=0}^{p} a_{r i j} t^{r+i}(\log t)^{j}
$$

where $r=r_{1}, \cdots, r_{k} \in \mathbb{Q}>-1$ and $p=\operatorname{dim} H^{0}\left(X_{t}, \Omega_{X_{t}}^{n}\right)$.

Proof of Theorem 0.1. Let $\|\cdot\|_{Q}$ be the Quillen metric of $\lambda\left(\mathcal{O}_{X}\right)$ relative to $g_{X / S}$. By the Definition 1.1 and Proposition 8.2, there exists $f \in C^{\infty}(S)$ such that

$$
\|\sigma\|_{Q}^{2}(t)=\exp (f(t)) \tau\left(X_{t}\right) \operatorname{det}\left({ }^{t} \Omega(t) \Lambda \bar{\Omega}(t)\right)^{(-1)^{n+1}}
$$

Let $g_{X}^{\prime}$ be a Kähler metric of $X$ such that $g_{X}^{\prime}$ is Euclidean flat on a neighborhood of Sing $X_{0}$ and $\left[g_{X}^{\prime}\right]=\left[g_{X}\right]$ in $H_{d R}^{2}(X, \mathbb{R})$. Let $g_{X / S}^{\prime}$ be the induced metric on $T X / S$ and $\|\cdot\|_{Q}^{\prime}$ the Quillen metric of $\lambda\left(\mathcal{O}_{X}\right)$ relative to $g_{X / S}^{\prime}$. By Main Theorem for $\left(\lambda\left(\mathcal{O}_{X}\right),\|\cdot\|_{Q}^{\prime}\right)$, there exists a smooth $(1,1)$-form $\chi$ on $S$ such that, in the sense of current,

$$
\frac{i}{2 \pi} \bar{\partial} \partial \log \left(\|\sigma\|_{Q}^{\prime}\right)^{2}=\frac{(-1)^{n+1}}{(n+2) !} \mu\left(X_{0}\right) \delta_{0}+\chi
$$

because $\pi_{*}\left(\operatorname{Td}\left(T X / S, g_{X / S}^{\prime}\right)\right)^{(1,1)}$ is a smooth $(1,1)$-form on $S$ by Proposition 2.1, which together with Theorem 1.2 and Theorem 3.1 (1) yields

$$
\log \|\sigma\|_{Q}^{2}(t)=\frac{(-1)^{n}}{(n+2) !} \mu\left(X_{0}\right) \log |t|^{2}+F(t)
$$

where $F \in C^{\alpha}(S)$ with some $\alpha>0$. Moreover $F(t)$ admits the following asymptotic expansion:

$$
F(t) \sim \sum_{r=r_{1}, \cdots, r_{l}} \sum_{i, j \geq 0} \sum_{k=0}^{n} a_{r i j k}|t|^{r} t^{i} \bar{t}^{j}(\log |t|)^{k}
$$


where $r_{1}, \cdots, r_{l} \in \mathbb{Q}_{+}$, and so does $\exp (F(t))$. The theorem follows from (8.8), (8.10), (8.11) and Proposition 8.3.

THEOREM 8.1. Suppose that Sing $X_{0}$ consists of rational singularities. Then, there exists a constant $A$ such that

$$
\lim _{t \rightarrow 0} \frac{\tau\left(X_{t}\right)}{|t|^{2 \frac{(-1)^{n}}{(n+2) !} \mu\left(\operatorname{Sing} X_{0}\right)}}=\exp (A) .
$$

Proof. From the rationality of Sing $X_{0}$, it follows that any $\omega_{i}(0)$ is square integrable over $X_{0}$ (cf. $\left.[\mathrm{Bu}]\right)$ and $\operatorname{det}\left(\int_{X_{0}} \omega_{i}(0) \wedge \bar{\omega}_{j}(0)\right) \neq 0$ because $\left\{\omega_{1}(0), \cdots, \omega_{m}(0)\right\}$ is a basis of $H^{0}\left(X_{0}, \Omega_{X_{0}}^{n}\right)$ over $\mathbb{C}$. Therefore, by Theorem 0.1 , it is enough to show that

$$
\lim _{t \rightarrow 0} \int_{X_{t}} \omega_{i}(t) \wedge \bar{\omega}_{j}(t)=\int_{X_{0}} \omega_{i}(0) \wedge \bar{\omega}_{j}(0)
$$

For simplicity, we assume Sing $X_{0}=\{o\}$. Let $\left(U,\left(z_{0}, \cdots, z_{n}\right)\right)$ be a coordinate neighborhood around $o$ and $f \in \mathcal{O}(U)$ the local defining equation of $\left(X_{0}, o\right)$, i.e., $X_{0} \cap U=\{z \in U ; f(z)=0\}$. As $\pi$ is of maximal rank on $X-U$, it is clear

$$
\lim _{t \rightarrow 0} \int_{X_{t}-U} \omega_{i}(t) \wedge \bar{\omega}_{j}(t)=\int_{X_{0}-U} \omega_{i}(0) \wedge \bar{\omega}_{j}(0)
$$

By the definition of relative canonical form, there exists $\eta_{i} \in \mathcal{O}(U)$ such that $d f \wedge \omega_{i}=$ $\eta_{i} d z_{0} \wedge \cdots \wedge d z_{n}$.

Let $p: Y \rightarrow X$ be a good embedded resolution of $f, Y_{0}$ the proper transform of $X_{0}$ and $p^{-1}(0)=E_{1} \cup \cdots \cup E_{k}$ the exceptional divisors such that $Y_{0} \cup E_{1} \cup \cdots E_{k}$ is a divisor with normal crossing on $Y$. Let $Y_{t}=p^{-1}\left(X_{t}\right)$. Since Sing $X_{0}$ is rational, it follows from [v.S-St, (3.1) Theorem] that

$$
p^{*}\left(\frac{d f}{f}\right) \wedge p^{*} \omega_{i}=p^{*}\left(\eta_{i} \frac{d z_{0} \wedge \cdots \wedge d z_{n}}{f}\right) \in \Omega_{p^{-1}(U)}^{n+1}\left(\log Y_{0}\right) .
$$

Let $x \in Y_{0}$, and $\left(V,\left(w_{0}, \cdots, w_{n}\right)\right)$ be a local coordinates around $x$ such that $Y_{0} \cap V=$ $\left\{w \in V ; w_{0}=0\right\}$ and $p^{*} f(w)=w_{0} w_{1}^{d_{1}} \cdots w_{n}^{d_{n}}\left(d_{i} \in \mathbb{Z}_{\geq 0}\right)$. By (8.14), there exists $a_{i}(w) \in \mathcal{O}(V)$ such that

$$
\left.p^{*}\left(\eta_{i} \frac{d z_{0} \wedge \cdots \wedge d z_{n}}{f}\right)\right|_{V}=a_{i}(w) \frac{d w_{0}}{w_{0}} \wedge d w_{1} \wedge \cdots \wedge d w_{n}
$$

By (8.14) again, we get

$$
p^{*} \omega_{i}=a_{i}(w) d w_{1} \wedge \cdots \wedge d w_{n}+p^{*} d f \wedge \xi_{i}
$$

for some $\xi_{i} \in \Omega_{V}^{n-1}\left(* Y_{0}+* E_{1}+\cdots+* E_{k}\right)$ which yields

$$
\begin{aligned}
\lim _{t \rightarrow 0} \int_{Y_{t} \cap V} p^{*} \omega_{i}(t) \wedge \overline{p^{*} \omega_{j}(t)} & =\int_{Y_{0} \cap V} a_{i}(w) \overline{a_{j}(w)} d w_{1} \wedge \cdots \wedge d w_{n} \wedge d \bar{w}_{1} \wedge \cdots \wedge d \bar{w}_{n} \\
& =\int_{Y_{0} \cap V} p^{*} \omega_{i}(0) \wedge \overline{p^{*} \omega_{j}(0)} .
\end{aligned}
$$

Similarly, for any $x \in E_{i}$, there is a neighborhood W such that

$$
\lim _{t \rightarrow 0} \int_{Y_{t} \cap W} p^{*} \omega_{i}(t) \wedge \overline{p^{*} \omega_{j}(t)}=\int_{Y_{0} \cap W} p^{*} \omega_{i}(0) \wedge \overline{p^{*} \omega_{j}(0)}
$$


which together with (8.13) and (8.17) yields (8.12).

REMARK. By (8.12), we find that -1 is a simple root of the $b$-function for IHS and any other root is strictly smaller than -1 .

\section{REFERENCES}

[Ba] BARlet, D., Développement asymptotique des fonctions obtenues par intégration dans la fibre, Invent. Math., 68 (1982), pp. 129-174.

[B1] Bismut, J.-M., Superconnection currents and complex immersions, Invent. Math., 99 (1990), pp. 59-113.

[B2] BISMUT, J.-M., Quillen metrics and singular fibers in arbitrary reletive dimension, preprint (1994).

[B-B] Bismut, J.-M., Bost, J.-B., Fibrés déterminants, métrique de Quillen et dégénérescence des corbes, Acta Math., 165 (1990), pp. 1-103.

[B-G-S] Bismut, J.-M., Gillet, H., Soulé, C., Analytic torsion and holomorphic determinant bundles I, II,III, Commun. Math. Phys., 115 (1988), pp. 49-78, 79-126, 301-351.

[B-L] Bismut, J.-M., Lebeau, G., Complex immersions and Quillen metrics, Publ. Math. IHES, 74 (1991), pp. 1-297.

[Bu] Burns, D., On rational singularities in dimension > 2, Math. Ann., 211 (1974), pp. 237244.

[D-L-S] Demailly, J.-P., Lempert, L., Shiffman, B., Algebraic approximations of holomorphic maps from Stein domains to projective manifolds, Duke Math. J., 76 (1994), pp. 333363.

[F] Faltings, G., Lectures on the arithmetic Riemann-Roch theorem, Ann. of Math. Study, 127 (1992).

[Fa] Farber, M.S., Singularities of analytic torsion, J. Diff. Geom., 41 (1995), pp. 528-572.

[G-T] Gilberg, D. AND Trudinger, N.S., Elliptic partial differential equations of second order, Springer, Berlin, Heidelberg, New York, 1983.

[G] Grothendeck, A., Techniques de construction et théorem d'existence en géométrie algébrique IV: Les schémas de Hilbert, Séminaire Bourbaki, 13 (1960/61).

[K] KоваYASHI, S., Differential gometry of complex vector bundles, Iwanami Shoten Publishers, Tokyo, 1987.

[L] Lempert, L., Algebraic approximation in analytic geometry, Invent. Math., 121 (1995), pp. 335-354.

[Lo] LooiJenga, E.J.N., Isolated singular points on complete intersections, London Math. Soc. Lect. Note 77, Cambridge University Press.

[M] Malgrange, B., Integrales asymptotiques et monodromie, Ann. Sci. Ec. Norm. Sup., 7 (1974), pp. 405-430.

[Q] Quillen, D., Determinant of Cauchy-Riemann operators over a Riemann surface, Funt. Anal. Appl., 19 (1985), pp. 31-34.

[S] SoulÉ, C. ET AL., Lectures on Arakelov geometry, Cambridge University Press, Cambridge, 1992.

[St] Steenbrink, J.H.M., Mixed Hodge structures on the vanishing cohomology, Real and Complex Singularities, Oslo (1976), pp. 525-563.

[v.S-St] v.Straten, D., Steenbrink, J.H.M., Extendability of holomorphic differential forms near isolated hypersurface singularities, Abh. Math. Sem. Univ. Hamburg, 55 (1985), pp. 97110.

[T-T] Tong, Y.-L.-L., TSAI, I-H., Curvature of determinant bundles for degenerate families, Commun. Math. Phys., 171 (1995), pp. 589-606.

[W] Wolpert, S.A., Asymptotics of the spectrum and the Selberg zeta function on the space of Riemann surface, Commun. Math. Phys., 112 (1987), pp. 283-315.

[Y1] Yoshikawa, K.-I., Smoothing of isolated hypersurface singularities and the Quillen metric, preprint, Institut Fourier, Grenoble (1996).

[Y2] Yoshikawa, K.-I., Discriminant of theta divisors and Quillen metrics, preprint (1997). 